\title{
OPERATORS WITH SMOOTH FUNCTIONAL CALCULI
}

\author{
MATS ANDERSSON \& HÅKAN SAMUELSSON \& SEBASTIAN SANDBERG
}

\begin{abstract}
We introduce a class of (tuples of commuting) unbounded operators on a Banach space, admitting smooth functional calculi, that contains all operators of Helffer-Sjöstrand type and is closed under the action of smooth proper mappings. Moreover, the class is closed under tensor product of commuting operators. In general an operator in this class has no resolvent in the usual sense so the spectrum must be defined in terms of the functional calculus. We also consider invariant subspaces and spectral decompositions.
\end{abstract}

\section{INTRODUCTION}

In this paper we study unbounded operators on a Banach space $X$ that admit smooth functional calculi, although they do not necessarily have resolvents. Throughout this paper $X$ is a complex Banach space, $\mathrm{E}(X)$ is the space of bounded linear operators on $X$, and $e_{X}$ denotes the identity operator.

Let $a$ be a closed (densely defined) operator with real spectrum and with the property that for each compact set $K \subset \subset \mathbb{C}$ there are $N_{K}$ and $C_{K}$ such that

$$
\left\|\omega_{z-a}\right\| \leq C_{K}|\operatorname{Im} z|^{-N_{K}}, \quad z \in K \backslash \mathbb{R}
$$

where $\omega_{z-a}$ is the resolvent form $\omega_{z-a}=(z-a)^{-1} d z / 2 \pi i$. Then there is a continuous multiplicative mapping $[a]: \mathcal{D}(\mathbb{R}) \rightarrow \mathrm{E}(X)$, defined by

$$
[a](\phi)=\int \omega_{z-a} \wedge \bar{\partial} \tilde{\phi}
$$

where $\tilde{\phi}$ is an almost holomorphic extension to $\mathbb{C}$ of $\phi$ with compact support. This was done by Dynkin, 7], for bounded operators $a$ and for unbounded operators by Helffer and Sjöstrand, 10. If $a$ is bounded, $[a]$ acts on all smooth functions $\phi$ on $\mathbb{R}$ and it coincides with the holomorphic functional calculus if $\phi$ is holomorphic in a neighborhood of the spectrum. In general, $[a]$ has a continuous extension to the algebra $\mathcal{G}$ of all smooth functions on $\mathbb{R}$ that are holomorphic at infinity, in particular, to each $r_{z}(\xi)=1 /(z-\xi)$ for $z \in \mathbb{C} \backslash \mathbb{R}$, and $[a]\left(r_{z}\right)=(z-a)^{-1}$.

Date: September 7, 2018.

1991 Mathematics Subject Classification. 47A60; 47A13; 32A26. 
Conversely, it was proved in [3] that if there exists such a multiplicative mapping $[a]$ such that, in addition,

$$
\cup_{\phi \in \mathcal{D}(\mathbb{R})} \operatorname{Im}[a](\phi) \text { is dense, } \cap_{\phi \in \mathcal{D}(\mathbb{R})} \operatorname{Ker}[a](\phi)=\{0\},
$$

and $[a]$ extends continuously to $\mathcal{G}$, then there is a closed operator $a$ satisfying (1.1) and such that (1.2) holds, see Theorem 6.3 for the precise statement. However, in many cases there exists such a smooth functional calculus although the resolvent does not exist at all. For example, let $a$ be multiplication with $\xi \mapsto \xi\left(2+\sin \xi^{3}\right)$ on $X=H^{1}(\mathbb{R})$. Then the resolvent set is empty, but nevertheless $a$ admits a smooth functional calculus $\mathcal{D}(\mathbb{R}) \rightarrow \mathrm{E}(X)$, and (1.3) holds.

We take the existence of a smooth functional calculus as our starting point, and introduce the notion of a hyperoperator, (with respect to smooth functions). It is a multiplicative $\mathrm{E}(X)$-valued distribution $A$ on $\mathbb{R}$ such that (1.3) holds. This additional requirement means that $A(1)=e_{X}$ in a weak sense. The spectrum of $A$ is defined as the support of the distribution. A closable operator (tuple of commuting closable operators) defined on a dense subspace $D$ is a weak hyperoperator, who, if $a$ admits an $\mathcal{E}$ functional calculus with respect to $D$, i.e., a multiplicative continuous mapping $\mathcal{E}\left(\mathbb{R}^{n}\right) \rightarrow \mathrm{E}(D)$, where $\mathrm{E}(D)$ is the set of closable operators mappings $D \rightarrow D$. Roughly speaking this means that each $x \in D$ has real and compact local spectrum with respect to $D$. If $a$ is a who and $f$ is any smooth mapping then $f(a)$ is again a who. It turns out that for any hyperoperator $A$ there is an associated who $a$. If $f$ is proper, then the push-forward $B=f_{*} A$ of $A$ is a hyperoperator and $b=f(a)$ is the who associated to $B$. Conversely, a who $a$ is (or corresponds to) a hyperoperator if and only if for each $\phi \in \mathcal{D}\left(\mathbb{R}^{n}\right), \phi(a)$ extends to a bounded operator on $X$. Moreover, $a$ is bounded (extends to a bounded operator) if and only if for each $f \in \mathcal{E}\left(\mathbb{R}^{n}\right), f(a)$ extends to a bounded operator on $X$.

It is a well-known problem to find a suitable definition of commutativity for unbounded operators to get a reasonable theory. We will consider hyperoperators on $\mathbb{R}^{n}$ as well, with a completely analogous definition. For instance, if $A_{1}$ and $A_{2}$ are hyperoperators in $\mathbb{R}$, with associated whos $a_{1}$ and $a_{2}$, commuting in the functional calculus sense, then $A=A_{1} \otimes A_{2}$ is a new hyperoperator in $\mathbb{R}^{2}$, and $a=\left(a_{1}, a_{2}\right)$ is the associated who. However, it is not true that each hyperoperator in $\mathbb{R}^{2}$ appears in this way. Similar phenomena hold for the unbounded analogs of a commuting tuple of bounded operators that are studied in e.g., 12, 15], 22], and [23. This gives support for the idea that a reasonable notion of "commuting tuple of unbounded operators" must be considered as an object in its own. Weaker forms of commutativity of unbounded operators are studied in [17], [18], [19], and 20].

One can think of (1.2) as meaning that

$$
\bar{\partial} \omega_{z-a}=[a]
$$


where $[a]$ is the operator-valued distribution $\phi \mapsto \phi(a)$. For a general hyperoperator the resolvent form does not exist, but we present other solutions to (1.4) such that representations like (1.2) still holds.

\section{Contents}

\begin{tabular}{|lr|}
\hline 1. Introduction & 1 \\
\hline 2. Notation and some preliminaries & 3 \\
\hline 2.1. The Dvnkin-Helffer-Siöstrand functional calculus & 4 \\
\hline 2.2. Commuting bounded operators & 5 \\
\hline 3. Definition and basic properties & 6 \\
\hline 4. Weak hvperoperators & 8 \\
\hline 5. Spectrum of a hvperoperator & 13 \\
\hline 6. Representation bv pseudoresolvents & 15 \\
\hline 7. Temperate hvperoperators & 19 \\
\hline 8. Operators with ultradifferentiable functional calculus & 22 \\
\hline 9. Invariant subspaces and spectral decomposition & 24 \\
\hline 10. Non-commuting hyperoperators & 26 \\
\hline References & 27 \\
\hline
\end{tabular}

\section{Notation AND SOME PRELIMINARIES}

Any closed (densely defined) operator $a$ on $X$, has a well-defined resolvent set $\rho(a)$ which is an open (possibly empty) subset of the extended plane $\widehat{\mathbb{C}}$. The spectrum of $a$ is the set $\sigma(a)=\widehat{\mathbb{C}} \backslash \rho(a)$. Moreover, the operator $a$ is bounded if and only if its spectrum is contained in $\mathbb{C}$. For any automorphism $\phi(\zeta)$ of $\widehat{\mathbb{C}}$ such that $\phi^{-1}(\infty)$ is not in the point spectrum of $a, \phi(a)$ is a well-defined closed operator, and the spectral mapping property $\phi(\sigma(a))=\sigma(\phi(a))$ holds. The automorphism

$$
C(\zeta)=\frac{\zeta+i}{\zeta-i}, \quad C^{-1}(\tau)=i \frac{\tau+1}{\tau-1},
$$

maps $\widehat{\mathbb{R}}$ bijectively onto to the unit circle $\mathbb{T}$. It induces the Cayley transform which establishes a one-to-one correspondence between closed operators with spectrum contained in $\mathbb{R}$, and bounded operators $b$ with spectrum contained in $\mathbb{T}$ such that $b-e_{X}$ is injective.

If $a$ is a densely defined operator on $X$, then it is closable if there is a closed operator $a^{\prime}$ such that $a \subset a^{\prime}$, i.e., that the graph of $a$ is contained in the graph of $a^{\prime}$. In that case the closure of the graph of $a$ is the graph of a (closed) operator called the closure $\bar{a}$ of $a$. If $a$ has a bounded extension, then it is equal to $\bar{a}$. 
We let $H^{k}\left(\mathbb{R}^{n}\right)$ denote the Sobolev space consisting of all functions in $L^{2}\left(\mathbb{R}^{n}\right)$ such that all derivatives up to order $k$ belongs to $L^{2}\left(\mathbb{R}^{n}\right)$ as well.

2.1. The Dynkin-Helffer-Sjöstrand functional calculus. For any $\phi \in \mathcal{D}(\mathbb{R})$ one can find an extension $\tilde{\phi}$ to $\mathbb{C}$ such that

$$
\bar{\partial} \tilde{\phi}(\zeta)=\varnothing\left(|\operatorname{Im} \zeta|^{\infty}\right) ;
$$

such a $\tilde{\phi}$ is called an almost holomorphic extension of $\phi$. Moreover, if $\tilde{K}$ is a complex neighborhood of $\operatorname{supp} \phi$, one may assume that $\tilde{\phi}$ has support in $\tilde{K}$. Now let $a$ be a closed operator with real spectrum such that (1.1) holds, such an operator will be referred to as an HS operator. Then clearly the integral in (1.2) converges, and it turns out to be independent of the choice of almost holomorphic extension. The multiplicativity follows from an application of the resolvent identity

$$
\frac{1}{w-a} \frac{1}{z-a}=\frac{1}{z-w} \frac{1}{w-a}+\frac{1}{w-z} \frac{1}{z-a} .
$$

It is easy to see that $[a]$ is continuous in the sense that $[a]\left(\phi_{j}\right) \rightarrow 0$ in operator norm if $\phi_{j} \rightarrow 0$ in $\mathcal{D}(\mathbb{R})$. It also follows that the support of $[a]$ coincides with $\sigma(a) \cap \mathbb{C}$. Moreover, we claim that

$$
[a](\xi \phi) x=[a](\phi) a x, \quad x \in \operatorname{Dom}(a) .
$$

This is of course well-known, but for further reference we sketch a proof. From the resolvent identity we have, assuming that $-i$ is outside the support of $\tilde{\phi}$,

$$
\begin{aligned}
& \int\left(\frac{1}{a+i}-\frac{1}{z+i}\right) \frac{d z}{z-a} \wedge \bar{\partial} \tilde{\phi}(z)= \\
& \quad \int \frac{d z}{(a+i)(z+i)} \wedge \bar{\partial} \tilde{\phi}(z)=-\int \bar{\partial}\left(\frac{d z}{(a+i)(z+i)} \wedge \tilde{\phi}(z)\right)=0,
\end{aligned}
$$

where the last equality follows from Stokes' theorem. Thus we have

$$
\frac{1}{a+i}[a](\phi)=[a](\phi) \frac{1}{a+i}=[a]\left(\frac{1}{\xi+i} \phi(\xi)\right) .
$$

Replacing $\phi(\xi)$ by $(\xi+i) \phi(\xi)$ we get

$$
\frac{1}{a+i}[a]((\xi+i) \phi)=[a]((\xi+i) \phi) \frac{1}{a+i}=[a](\phi) .
$$

If $x \in \operatorname{Dom}(a)$ we therefore have $[a]((\xi+i) \phi) x=[a](\phi)(a+i) x$, which implies (2.2).

Example 1. Let $a$ be a closed operator with spectrum equal to $\{\infty\}$. For instance one can take the inverse of the Volterra operator. Then clearly (1.1) holds, but the resulting multiplicative mapping $[a]$ is identically 0 . 
If $a_{1}, \ldots, a_{n}$ is a tuple of HS operators such that their resolvents (anti) commute, i.e., $\omega_{\zeta_{j}-a_{j}} \wedge \omega_{\zeta_{k}-a_{k}}=-\omega_{\zeta_{k}-a_{k}} \wedge \omega_{\zeta_{j}-a_{j}}$, for $\zeta_{j}, \zeta_{k} \in \mathbb{C} \backslash \mathbb{R}$, then $[a]=\left[a_{1}\right] \otimes\left[a_{2}\right] \cdots \otimes\left[a_{n}\right] \in \mathcal{D}^{\prime}\left(\mathbb{R}^{n}, \mathrm{E}(X)\right)$ is multiplicative. This follows by simple abstract considerations, but it can also be realized explicitly as

$$
[a](\phi)=\int \omega_{\zeta_{1}-a_{1}} \wedge \ldots \wedge \omega_{\zeta_{n}-a_{n}} \wedge \bar{\partial}_{\zeta_{n}} \ldots \bar{\partial}_{\zeta_{1}} \tilde{\phi}(\zeta)
$$

where $\tilde{\phi}$ is a special almost holomorphic extension to $\mathbb{C}^{n}$ with compact support as in [3], i.e., such that

$$
\bar{\partial}_{\zeta_{1}} \cdots \bar{\partial}_{\zeta_{m}} \tilde{\phi}(\zeta)=\varnothing\left(\left|\operatorname{Im} \zeta_{1}\right|^{\infty} \cdots\left|\operatorname{Im} \zeta_{m}\right|^{\infty}\right) .
$$

2.2. Commuting bounded operators. Let $a=\left(a_{1}, \ldots, a_{n}\right)$ be a commuting tuple of bounded operators on $X$. If the Taylor spectrum $\sigma(a)$ is contained in $\mathbb{R}^{n}$, then it coincides with the spectrum of $a$ with respect to the commutative Banach algebra $(a)$ generated by $a$. If the tuple $a$ has real spectrum, then we say that $a$ admits a smooth functional calculus if the real-analytic functional calculus $[a]: \varnothing\left(\mathbb{R}^{n}\right) \rightarrow \mathrm{E}(X)$ has a continuous extension to a mapping $[a]: \mathcal{E}\left(\mathbb{R}^{n}\right) \rightarrow \mathrm{\iota}(X)$. Since $C^{\omega}\left(\mathbb{R}^{n}\right)$ is dense in $\mathcal{E}\left(\mathbb{R}^{n}\right)$, the extension is then unique and multiplicative, and in fact it extends to $\mathcal{E}(\sigma(a)) \rightarrow \mathrm{E}(X)$. The existence of such an extension is equivalent to that $\exp ($ iat $)$ has polynomial growth in $t \in \mathbb{R}^{n}$, see, e.g., [1; it is also equivalent to that the resolvent satisfies

$$
\left\|\omega_{z-a}\right\| \leq C|\operatorname{Im} z|^{-M}
$$

for some $M>0$.

If $a$ has non-real (Taylor) spectrum $\sigma(a)$, then there is in general no unique extension of the holomorphic functional calculus. For instance, let $b$ be a nilpotent operator and let $A(\phi)=\tilde{\phi}(b, 0)$ and $B(\phi)=\tilde{\phi}(b, b)$ respectively, where $\tilde{\phi}(z, \bar{z})=\phi(z)$ for real-analytic $\phi$ (only a finite Taylor expansion is needed). Then $A$ and $B$ extend to two different multiplicative mappings $\mathcal{E}(\mathbb{C}) \rightarrow \mathrm{E}(X)$ which both extend the holomorphic functional calculus. In general, a possible smooth functional calculus is uniquely determined by the image of $\bar{z}$ (or $\bar{z}_{j}$ if we have an $n$-tuple of commuting operators). In our situation the bounded (tuples of) operators that appear are like $b=A(\phi)$ for a possibly complexvalued $\phi$, and then we have a natural conjugated operator, namely $b^{*}=A(\bar{\phi})$. A smooth functional calculus for such an operator $b$ is then understood to map $\bar{z}$ to $b^{*}$. If $f(z)=\tilde{f}(z, \bar{z})=\hat{f}(\operatorname{Re} z, \operatorname{Im} z)$, then $f(b)=\tilde{f}\left(b, b^{*}\right)=\hat{f}\left(\left(b+b^{*}\right) / 2,\left(b-b^{*}\right) / 2 i\right)$, and therefore we can reduce to the case of real-valued functions $\phi$.

We conclude this section with the following useful observation.

Lemma 2.1. If $A$ is a linear and multiplicative mapping $\mathcal{D}(\mathbb{R}) \rightarrow \mathcal{L}(X)$ then, for any $\chi \in \mathcal{D}(\mathbb{R}), z \mapsto A(\chi(\xi) /(z-\xi))$ is strongly holomorphic in $\mathbb{C} \backslash \mathbb{R}$. 
Proof. Let $\tilde{\chi} \in \mathcal{D}(\mathbb{R})$ be identically 1 on $\operatorname{supp} \chi$. From linearity and multiplicativity we get

$$
A\left(\frac{\chi(\xi)}{z-\xi}\right)=A\left(\frac{\chi(\xi)}{z_{0}-\xi}\right)-\left(z-z_{0}\right) A\left(\frac{\chi(\xi)}{z-\xi}\right) A\left(\frac{\tilde{\chi}(\xi)}{z_{0}-\xi}\right) .
$$

Letting $\left\|A\left(\chi(\xi) /\left(z_{0}-\xi\right)\right)\right\|=C$ and $\left\|A\left(\tilde{\chi}(\xi) /\left(z_{0}-\xi\right)\right)\right\|=\tilde{C}$ we see that

$$
\|A(\chi(\xi) /(z-\xi))\| \leq C+\left|z-z_{0}\right| \tilde{C}\|A(\chi(\xi) /(z-\xi))\|
$$

and so $\|A(\chi(\xi) /(z-\xi))\| \leq C /\left(1-\left|z-z_{0}\right| \tilde{C}\right)$. Thus $\| A(\chi(\xi) /(z-$ $\xi)) \|$ is locally uniformly bounded in $z$. From (2.6) it now follows that $A(\chi(\xi) /(z-\xi))$ is strongly continuous at $z_{0}$. With this fact in mind it follows immediately from (2.6) that

$$
\frac{1}{z-z_{0}}\left(A\left(\frac{\chi(\xi)}{z-\xi}\right)-A\left(\frac{\chi(\xi)}{z_{0}-\xi}\right)\right) \rightarrow-A\left(\frac{\chi(\xi)}{\left(z_{0}-\xi\right)^{2}}\right), \quad z \rightarrow z_{0},
$$

in operator norm.

\section{Definition AND BASIC PROPERTIES}

We say that a linear mapping $A: \mathcal{D}\left(\mathbb{R}^{n}\right) \rightarrow \mathrm{E}(X)$ is continuous, $A \in \mathcal{D}^{\prime}\left(\mathbb{R}^{n}, \mathrm{E}(X)\right)$, if $A\left(\phi_{j}\right) \rightarrow 0$ in operator norm when $\phi_{j} \rightarrow 0$ in $\mathcal{D}\left(\mathbb{R}^{n}\right)$. As for ordinary distributions it follows immediately that $A$ has finite order on compact subsets, i.e., for any compact $K \subset \mathbb{R}^{n}$ there is a constant $C_{K}$ and a non-negative integer $M_{K}$ such that

$$
\|A(\phi)\| \leq C_{K} \sum_{|\alpha| \leq M_{K}} \sup _{K}\left|\partial^{\alpha} \phi\right|
$$

for all $\phi \in \mathcal{D}\left(\mathbb{R}^{n}\right)$ with support in $K$.

Definition 1. A continuous multiplicative mapping $A: \mathcal{D}\left(\mathbb{R}^{n}\right) \rightarrow \mathrm{E}(X)$ is a hyperoperator on $\mathbb{R}^{n}, A \in H_{\mathcal{D}\left(\mathbb{R}^{n}\right)}(X)$, if

$$
\text { (i) } D_{A}=\cup \operatorname{Im} A(\phi) \text { is dense in } X, \quad \text { and }
$$

$$
\text { (ii) } \quad N=\cap \operatorname{Ker} A(\phi)=\{0\} \text {. }
$$

If $a$ is an HS operator such that $[a]$ satisfies $(i)$ and $(i i)$, then $[a]$ is a hyperoperator. If $a$ is bounded (or a commuting tuple of bounded operators), then $[a](\phi)=\phi(a)$. It is readily checked that the operator (tuple of operators) $0_{X}$ gives rise to the hyperoperator $\left[0_{X}\right]$, defined by $\left[0_{X}\right](\phi)=\phi(0) e_{X}$. In the same way, $\left[e_{X}\right](\phi)=\phi(1) e_{X}$.

Remark 1 . Let $A: \mathcal{D}\left(\mathbb{R}^{n}\right) \rightarrow \mathrm{E}(X)$ be a continuous multiplicative mapping. If $A$ has compact support, i.e., $A$ has a continuous extension to $\mathcal{E}\left(\mathbb{R}^{n}\right)$, then $(i)$ and $(i i)$ hold if and only if $A(1)=e_{X}$. In fact, let $\chi_{N}$ be a sequence in $\mathcal{D}\left(\mathbb{R}^{n}\right)$ that tends to 1 in $\mathcal{E}\left(\mathbb{R}^{n}\right)$. If now $A(1)=e_{X}$, then for any $x \in X$ we have that $x=A(1) x=\lim A\left(\chi_{N}\right) x$, and hence $(i)$ holds. In the same way, if $A\left(\chi_{N}\right) x=0$ for all $N$, then $x=A(1) x=0$ so that (ii) holds as well. Conversely, if $x \in D_{A}$, then $x=A(\phi) z$ and 
therefore $A(1) x=A(1) A(\phi) z=A(1 \cdot \phi) z=A(\phi) z=x$. If $D_{A}$ is dense it follows that $A(1)=e_{X}$. Therefore it is natural to think of $(i)$ and (ii) as a weak form of saying that $A(1)=e_{X}$.

We say that $\chi_{N} \in \mathcal{D}\left(\mathbb{R}^{n}\right)$ is an exhausting sequence if $0 \leq \chi_{N} \leq 1$, $\chi_{N} \nearrow 1$, and the compact sets $K_{N}=\left\{\chi_{N}=1\right\}$ form an exhausting sequence of compact sets; i.e., $K_{N} \subset \operatorname{int}\left(K_{N+1}\right)$ and $\cup K_{N}=\mathbb{R}^{n}$.

Lemma 3.1. Suppose that $\chi_{N}$ is an exhausting sequence in $\mathbb{R}^{n}$ and $A \in H_{\mathcal{D}\left(\mathbb{R}^{n}\right)}(X)$. Then $\cup \operatorname{Im} A\left(\chi_{N}\right)=D_{A}$.

Proof. If $\phi \in \mathcal{D}\left(\mathbb{R}^{n}\right)$, then $\chi_{N} \phi=\phi$ if $N$ is large enough, and therefore

$$
A\left(\chi_{N}\right) A(\phi)=A\left(\chi_{N} \phi\right)=A(\phi),
$$

which shows that $A\left(\chi_{N}\right)$ is the identity on $\operatorname{Im} A(\phi)$. Thus $\operatorname{Im} A\left(\chi_{N}\right) \supset$ $\operatorname{Im} A(\phi)$.

Proposition 3.2. Assume that $A_{1}$ and $A_{2}$ are hyperoperators in $\mathbb{R}^{n}$ and $\mathbb{R}^{m}$, respectively, and that they are commuting, i.e.,

$$
A_{1}(\phi) A_{2}(\psi)=A_{2}(\psi) A_{1}(\phi), \quad \phi \in \mathcal{D}\left(\mathbb{R}^{n}\right), \quad \psi \in \mathcal{D}\left(\mathbb{R}^{m}\right) .
$$

Then $A=A_{1} \otimes A_{2}$ is a hyperoperator in $\mathbb{R}^{n+m}$ and $D_{A}=D_{A_{1}} \cap D_{A_{2}}$.

In particular it follows that $D_{A_{1}} \cap D_{A_{2}}$ is dense as soon as $A_{1}$ and $A_{2}$ are commuting.

Proof. The tensor product $A$ is defined as usual for distributions; thus $A(\phi \otimes \psi)=A_{1}(\phi) A_{2}(\psi)$, and it is extended to $\mathcal{D}\left(\mathbb{R}^{n+m}\right)$ by linearity and continuity. The assumption on commutativity implies that $A$ is multiplicative. If $0=A(\phi \otimes \psi) x=A_{1}(\phi) A_{2}(\psi) x$ for all $\phi$ and $\psi$ it follows from condition ( $i i)$ for $A_{1}$ and $A_{2}$ that $x=0$. Thus $(i i)$ holds for $A$. Given $x \in X$ we can find $y$ and $\phi$ such that $\left\|x-A_{1}(\phi) y\right\|<\epsilon / 2$. In the same way we can find $z$ and $\psi$ such that $\left\|y-A_{2}(\psi) z\right\|<\epsilon /\left(2\left\|A_{1}(\phi)\right\|\right)$. It follows that $\|x-A(\phi \otimes \psi) z\|<\epsilon$. Thus $D_{A}$ is dense in $X$. On the other hand, since $\chi_{N} \otimes \chi_{M}^{\prime}$ is an exhausting sequence in $\mathbb{R}^{n+m}$ if $\chi_{N}$ and $\chi_{M}^{\prime}$ are exhausting sequences in $\mathbb{R}^{n}$ and $\mathbb{R}^{m}$, respectively, it follows that $x \in D_{A}$ if and only if $A\left(\chi_{N} \otimes \chi_{M}\right) x=x$ for sufficiently large $N$ and $M$, and this in turn holds if and only if $x \in D_{A_{1}} \cap D_{A_{2}}$.

If a hyperoperator $A$ in $\mathbb{R}^{n+m}$ is the tensor product $A_{1} \otimes A_{2}$ of two commuting, multiplicative $\mathcal{L}(X)$-valued distributions in $\mathbb{R}^{n}$ and $\mathbb{R}^{m}$, then each $A_{j}$ is indeed a hyperoperator. In fact, since $\chi_{N} \otimes \chi_{M}^{\prime}$ is exhausting in $\mathbb{R}^{n+m}, \cup \operatorname{Im} A\left(\chi_{N} \otimes \chi_{M}^{\prime}\right)=\cup \operatorname{Im} A_{1}\left(\chi_{N}\right) A_{2}\left(\chi_{M}^{\prime}\right)=$ $\cup \operatorname{Im} A_{2}\left(\chi_{M}^{\prime}\right) A_{1}\left(\chi_{N}\right)$ is dense, so $A_{j}$ satisfy condition $(i)$. If $A_{j}(\phi) x=0$ for all $\phi \in \mathcal{D}\left(\mathbb{R}^{n}\right)$, then $A\left(\phi \otimes \phi^{\prime}\right) x=0$ for all $\phi, \phi^{\prime}$. Therefore $A(\psi) x=0$ for all $\psi \in \mathcal{D}\left(\mathbb{R}^{n+m}\right)$, so $x=0$. Hence $A_{j}$ satisfies $(i i)$.

Proposition 3.3. If $A \in H_{\mathcal{D}\left(\mathbb{R}^{n}\right)}(X)$ and $f \in \mathcal{E}\left(\mathbb{R}^{n}, \mathbb{R}^{m}\right)$ is a proper mapping, then the push-forward $B=f_{*} A \in \mathcal{D}^{\prime}\left(\mathbb{R}^{m}, E(X)\right)$ is a hyperoperator, and $D_{B}=D_{A}$. 
Proof. Since $f$ is proper, $f^{*}: \mathcal{D}\left(\mathbb{R}^{m}\right) \rightarrow \mathcal{D}\left(\mathbb{R}^{n}\right)$ and hence $f_{*} A$, defined by $f_{*} A(\phi)=A\left(f^{*} \phi\right)=A(\phi \circ f)$, is a multiplicative distribution. If $\chi_{N}$ is an exhausting sequence in $\mathbb{R}^{m}$, since $f$ is proper, then $\chi_{N} \circ f$ is an exhausting sequence in $\mathbb{R}^{n}$. Therefore,

$$
D_{B}=\cup_{N} \operatorname{Im} f_{*} A\left(\chi_{N}\right)=\cup_{N} \operatorname{Im} A\left(\chi_{N} \circ f\right)=D_{A}
$$

according to Lemma 3.1. Thus $f_{*} A$ satisfies $(i)$. Finally, suppose that $f_{*} A(\psi) y=0$ for all $\psi \in \mathcal{D}\left(\mathbb{R}^{m}\right)$. For fixed $\phi \in \mathcal{D}\left(\mathbb{R}^{n}\right)$ and large $N$, then

$$
A(\phi) y=A\left(\phi\left(\chi_{N} \circ f\right)\right) y=A(\phi) A\left(\chi_{N} \circ f\right) y=0,
$$

and since $\phi$ is arbitrary, we conclude that $y=0$. Thus $f_{*} A$ is a hyperoperator.

It is easy to check that any hyperoperator $A$ extends to a multiplicative mapping on the algebra $\mathcal{D}\left(\mathbb{R}^{n}\right) \oplus \mathbb{C}$ of smooth functions that are constant outside some compact set, just by letting $A(h)=$ $h(\infty) e_{X}+A(h-h(\infty))$. If $\phi$ has compact support, then $h=f \circ \phi$ is in this algebra, and therefore we have

Proposition 3.4. Assume that $A \in H_{\mathcal{D}\left(\mathbb{R}^{n}\right)}(X)$ and $\phi \in \mathcal{D}\left(\mathbb{R}^{n}, \mathbb{R}^{m}\right)$. Then the bounded operator $\phi(a)=A(\phi)$ admits a $\mathcal{E}$-functional calculus that extends the holomorphic (real-analytic) functional calculus, defined by $f \mapsto f(0) e_{X}+A(f \circ \phi-f(0))$.

\section{WEAK HYPEROPERATORS}

We shall now see that for each hyperoperator $A$ there is an associated closable operator $a$ on $D_{A}$. We will use the operator $a$ to model the definition of a weak hyperoperator, see Definition 2 below.

Let $A$ be a hyperoperator in $\mathbb{R}^{n}$ and let $f: \mathbb{R}^{n} \rightarrow \mathbb{R}^{m}$ be any smooth mapping. If $x \in D_{A}$ and $x=A(\phi) y$ we define $f(a) x=A(f \phi) y$. If $\chi=1$ in a neighborhood of $\operatorname{supp} \phi$, then $f(a) x=A(f \chi \phi) y=$ $A(f \chi) A(\phi) y=A(f \chi) x$; thus $f(a) x=A(f \chi) x$ and in particular $f(a)$ is a well-defined densely defined operator. Also observe that if $\phi \in \mathcal{D}\left(\mathbb{R}^{n}\right)$, then $\phi(a) x=A(\phi) x$ for all $x \in D_{A}$.

For any $x \in X$ we let $\sigma_{x}(A)$ be the support of the $X$-valued distribution $\phi \mapsto A(\phi) x$; this is the local spectrum at $x$. If $K \subset \mathbb{R}^{n}$ is compact, we let

$$
D_{A, K}=\left\{x \in X ; \sigma_{x}(A) \subset K\right\} .
$$

It is readily checked that $D_{A}=\cup_{K} D_{A, K}$.

Proposition 4.1. Assume that $A$ is a hyperoperator in $\mathbb{R}^{n}$.

(a) If $f \in \mathcal{E}\left(\mathbb{R}^{n}, \mathbb{R}\right)$, then $f(a)$ maps $D_{A} \rightarrow D_{A}$, and if $g \in \mathcal{E}\left(\mathbb{R}^{n}, \mathbb{R}\right)$, then $g(a) f(a)=(f g)(a)$ on $D_{A}$.

(b) If $f \in \mathcal{E}\left(\mathbb{R}^{n}, \mathbb{R}^{m}\right)$, then $f(a)$ is a closable operator (tuple of operators). 
(c) If $f_{k} \rightarrow f$ in $\mathcal{E}\left(\mathbb{R}^{m}, \mathbb{R}^{p}\right)$, then $f_{k}(a) x \rightarrow f(a) x$ for all $x \in D_{A}$.

(d) If $x_{j} \in D_{A, K}$ for some fixed compact set $K$ and $x_{j} \rightarrow x$ in $X$, then $f(a) x_{j} \rightarrow f(a) x$.

Proof. If $x \in D_{A}$ and $y=f(a) x$, then $y=A(\chi f) x$ for an appropriate $\chi$ and hence by definition $y \in D_{A}$. Moreover,

$$
\begin{aligned}
g(a) f(a) x=g(a) A(\chi f) x=A(\tilde{\chi} g) A(\chi f) x=A(\tilde{\chi} \chi f g) x & = \\
A(\chi f g) x & =(f g)(a) x,
\end{aligned}
$$

if $A(\chi) x=x$ and $\tilde{\chi}=1$ on the support of $\chi$. Thus (a) holds.

We can always take the closure of the graph of $f(a)$ in $X^{n} \times X^{m}$. If $x_{k} \in D_{A}, x_{k} \rightarrow x$, and $f(a) x_{k} \rightarrow y$, then for any $\psi \in \mathcal{D}\left(\mathbb{R}^{n}\right)$, $A(\psi) f(a) x_{k} \rightarrow A(\psi) y$; but also $A(\psi) f(a) x_{k}=A(\psi f) x_{k} \rightarrow A(\psi f) x$, so $A(\psi) y=A(\psi f) x$. Because of condition (ii) we have that $y$ is then uniquely determined by $x$, and hence the closure is a graph. Thus $(b)$ is proved.

Given $x \in D_{A}$, take $\chi$ such that $A(\chi) x=x$. Since $f_{k} \chi \rightarrow f \chi$ in $\mathcal{D}\left(\mathbb{R}^{n}\right)$, we have that $f_{k}(a) x=A\left(f_{k} \chi\right) x \rightarrow A(\chi f) x=f(a) x$. Thus $(c)$ holds. For the last statement, observe that $A(\chi) x_{k}=x_{k}$ if $\chi=1$ in a neighborhood of $K$. Hence $f(a) x_{k}=A(f \chi) x_{k} \rightarrow A(f \chi) x=f(a) x$.

Notice that if $\phi \in \mathcal{D}\left(\mathbb{R}^{n}\right)$, then the closure of $\phi(a)$ is equal to the bounded operator $A(\phi)$. Moreover, the closure of $1(a)$ is equal to $e_{X}$ and the closure of $0(a)$ is equal to $0_{X}$. Applying Proposition 4.1 to the mapping $f(\xi)=\xi$, we find that $a$ has a meaning as a densely defined closable operator (tuple of closable operators $a_{j}=f_{j}(a)$, where $f_{j}(\xi)=\xi_{j}$, that commute on $\left.D_{A}\right)$.

In view of this proposition it is natural to introduce a special class of densely defined linear operators. If $D$ is a dense subspace, let $\mathrm{E}(D)$ be the set of closable linear operators $D \rightarrow D$.

Definition 2. Let $c=(c, D)$ be a linear operator mapping the dense subspace $D$ of $X$ into itself. Moreover, assume that $c$ is closable, and that there is a linear and multiplicative mapping $\mathcal{E}\left(\mathbb{R}^{n}\right) \rightarrow \mathrm{E}(D)$, that extends the trivial one on polynomials, and such that $h_{k}(c) x \rightarrow h(c) x$, for $x \in D$ if $h_{k} \rightarrow h$ in $\mathcal{E}\left(\mathbb{R}^{n}\right)$. Then we say that $c$, or rather $(c, D)$, is a weak hyperoperator, a who.

The sum of two closable operators is not necessarily closable (so $\mathrm{E}(D)$ is not a space), so part of the requirement is that each polynomial $p(c)$ in $c$ is closable. Moreover, since the polynomials are dense in $\mathcal{E}$ the extension to $\mathcal{E}\left(\mathbb{R}^{n}\right) \rightarrow \mathcal{L}(D)$ is unique if it exists.

Assume that $A$ is a hyperoperator and $f$ is any smooth mapping. If $h \in \mathcal{E}\left(\mathbb{R}^{m}, \mathbb{R}^{p}\right)$ then we can define $h(f(a))=(h \circ f)(a)$ on $D_{A}$. Therefore $\left(a, D_{A}\right)$ as well as $\left(f(a), D_{A}\right)$ are whos. Also notice that if $f$ is proper, $B=f_{*} A$, and $a$ and $b$ are the associated whos, then $b=f(a)$. 
We say that a who $c=(c, D)$ is (extendable to) a hyperoperator $A$ if $D \subset D_{A}$ and $\phi(c) x=A(\phi) x$ for $x \in D$. If such an $A$ exists it is unique in view of Proposition 4.2 below. In the sequel we will therefore often talk about the hyperoperator $a$, meaning that $a$ is the who associated to some hyperoperator $A$.

Proposition 4.2. Suppose that $A$ and $A^{\prime}$ are in $H_{\mathcal{D}\left(\mathbb{R}^{n}\right)}(X)$ and that $D_{A} \cap D_{A^{\prime}}$ is dense. Moreover, assume that there is a dense subspace $D$ of $D_{A} \cap D_{A^{\prime}}$ such that $a_{j}=a_{j}^{\prime}$ on $D$ and map $D \rightarrow D$. Then $A=A^{\prime}$.

Proof. If $x \in D$ and $\chi$ is identically 1 on a large enough set, then

$$
A\left(\xi_{j} \chi\right) x=a_{j} x=a_{j}^{\prime} x=A^{\prime}\left(\xi_{j} \chi\right) x .
$$

Moreover, if $\tilde{\chi}$ is 1 in a neighborhood of $\operatorname{supp} \phi$, (recall that $x \in D$ implies that $a_{j} x \in D$ )

$$
\begin{aligned}
& A\left(\xi_{k} \xi_{j} \chi\right) x=A\left(\xi_{k} \xi_{j} \chi \tilde{\chi}\right) x=A\left(\xi_{k} \chi\right) A\left(\xi_{j} \tilde{\chi}\right) x= \\
& \quad A\left(\xi_{k} \chi\right) a_{j} x=a_{k} a_{j} x=a_{k}^{\prime} a_{j}^{\prime} x=\ldots=A^{\prime}\left(\xi_{k} \xi_{j} \chi\right) x,
\end{aligned}
$$

and so on, so we get $A(p \chi) x=A^{\prime}(p \chi) x$ for all polynomials $p$. If $\phi$ is a test function it follows by the Weierstrass approximation theorem that $A(\phi) x=A^{\prime}(\phi) x$, and hence $A(\phi)=A^{\prime}(\phi)$ since $D$ is dense.

Corollary 4.3. If $A$ is a hyperoperator and $f$ is proper, then $f_{*} A=$ $\left[0_{X}\right]$ if and only if $f(a)=0$. In particular, $A=\left[0_{X}\right]$ if and only if $a=0$.

In fact, if $B=f_{*} A$, then $b=f(a)$, so $b x=0=0_{X} x$ for all $x \in D_{B}=$ $D_{A}$. Hence, by the previous proposition, $B=\left[0_{X}\right]$.

Corollary 4.4. If $A, A^{\prime}$ are commuting hyperoperators and $a=a^{\prime}$ on $D_{A} \cap D_{A^{\prime}}$, then $A=A^{\prime}$.

This is just because $D_{A} \cap D_{A^{\prime}}$ is dense if $A$ and $A^{\prime}$ commute, cf., Proposition 3.2 .

Assume that $A$ is a hyperoperator and let $h$ be smooth and constant outside a compact set. It is easily checked that the bounded operator $A(h)=h(\infty) e_{X}+A(h-h(\infty)$ is the closure of the densely defined operator $h(a)$. Therefore, cf., Proposition 3.4.

$$
f(A(\phi)) x=(f \circ \phi)(a) x, \quad x \in D_{A},
$$

for any smooth $f$ if $\phi$ has compact support.

Proposition 4.5. Let $a_{0}$ be an $H S$ operator such that $\left[a_{0}\right]$ satisfies $(i)$ and (ii) so that $A=\left[a_{0}\right]$ is a hyperoperator. If a is the associated who, then $\bar{a}=a_{0}$.

Proof. Since by assumption $a_{0}+i$ has a bounded inverse, we have that $\operatorname{Dom}\left(a_{0}\right)=\operatorname{Dom}\left(a_{0}+i\right)=\operatorname{Im}\left(a_{0}+i\right)^{-1}$. If $x \in D_{A}$, then $x=A(\chi) x$ 
so by (2.4)

$$
x=A(\chi) x=\frac{1}{a_{0}+i} A((\xi+i) \chi) x,
$$

and hence $x \in \operatorname{Dom}\left(a_{0}\right)$ and by (2.2), $a x=a_{0} x$. Thus $a \subset a_{0}$.

Now, if $x \in \operatorname{Dom}\left(a_{0}\right)$ there is some $y$ such that $x=\left(a_{0}+i\right)^{-1} y$. Take $y_{k} \in D_{A} \subset \operatorname{Dom}\left(a_{0}\right)$ such that $y_{k} \rightarrow y$. Then $x_{k}=\left(a_{0}+i\right)^{-1} y_{k}=$ $(a+i)^{-1} y_{k} \in D_{A}$ according to (2.3). Thus

$$
a x_{k}=a_{0} x_{k}=\frac{a_{0}}{a_{0}+i} y_{k} \rightarrow \frac{a_{0}}{a_{0}+i} y=a_{0} x,
$$

since $a_{0} /\left(a_{0}+i\right)$ is bounded. Therefore, $\left(x, a_{0} x\right)$ belongs to the closure of (the graph of) $a$.

It is now easy to see that there exist non-trivial hyperoperators.

Example 2. Let $a$ be the unbounded operator defined as multiplication with $\xi$ on $X=H^{1}\left(\mathbb{R}_{\xi}\right)$. It defines a hyperoperator $A=[a]$ and the associated who is $\left(a, D_{A}\right)$ where $D_{A}=\{x \in X ; \operatorname{supp} x \subset \subset \mathbb{R}\}$. The mapping $f(\xi)=\xi\left(2+\sin \xi^{3}\right): \mathbb{R} \rightarrow \mathbb{R}$ is proper and so $B:=f_{*} A$ is a hyperoperator with $D_{B}=D_{A}$. By definition $B(\phi)$ is multiplication with $\phi \circ f$. The who $b$ associated to $B$ is just multiplication with $f$ because if $x \in D_{B}$ and $\chi$ is chosen so that $\operatorname{supp} x \subset\{\chi \circ f=1\}$ then $b x=B(\xi \chi(\xi)) x=A(f \chi \circ f) x=f(a) x=f x$. We claim that $B$ is not $\left[b_{0}\right]$ for any HS operator $b_{0}$. If there were such a $b_{0}$, then by Proposition $4.5, \bar{b}=b_{0}$ and therefore there would be a bounded operator $c$ such that $c(\bar{b}+i) x=(\bar{b}+i) c x=x$ for all $x \in D_{B}=\operatorname{Dom}(b)$. However, then $c$ would have to be multiplication with $(f(\xi)+i)^{-1}$ on the image of $D_{B}$ under $\bar{b}+i$ which again is $D_{B}$, but this is impossible since multiplication with $(f(\xi)+i)^{-1}$ has no bounded extension to all of $X$.

Example 3. If $B$ is a hyperoperator in $\mathbb{R}^{2}$ then the associated who $b$ is equal to $\left(b_{1}, b_{2}\right)$ where $b_{j}=\pi_{j} b$ are whos as well. However it may happen that none of the $b_{j}$ are hyperoperators. Let $f_{1}(\xi)$ be equal to $\xi$ for $\xi>1$ and $\sin \xi^{2}$ for $\xi<-1$, and let $f_{2}(\xi)=-f_{1}(-\xi)$. Then $F=\left(f_{1}, f_{2}\right): \mathbb{R} \rightarrow \mathbb{R}^{2}$ is proper and therefore $B:=F_{*} A$ is a hyperoperator, if $A \in H_{\mathcal{D}(\mathbb{R})}\left(H^{1}(\mathbb{R})\right)$ is the hyperoperator that sends $\phi$ to multiplication with $\phi$. In this case $b_{1}=f_{1}(a)$ and $b_{2}=f_{2}(a)$. Now, $\phi\left(b_{j}\right)$ is multiplication with $\phi \circ f_{j}$ and this operator has in general no bounded extension to $H^{1}(\mathbb{R})$, so $b_{j}$ is not a hyperoperator. Take for instance $\phi \in \mathcal{D}(\mathbb{R})$ such that $\phi^{\prime}(\xi)=1$ for $-1 \leq \xi \leq 1$; then $\left(\phi \circ f_{j}\right)^{\prime}(\xi)$ is unbounded.

Example 4. Let $(M, \mu)$ be a finite measure space and let $h$ be a real or complex valued measurable function (tuple of functions) defined a.e. with respect to $\mu$. The operator defined as multiplication with $h$ on $L^{p}(M, \mu), 1 \leq p<\infty$, is then a hyperoperator and $\sigma(a)$ (see Section 5 ) is the essential range of $h$. Composing with smooth maps and/or taking 
tensor products will not take us outside this class of multiplication operators. By basic spectral theory any normal operator (tuple of normal commuting operators) can be viewed as such an operator (tuple of operators) on some $L^{2}(M, \mu)$. Therefore, our theory does not add anything to the usual theory of self-adjoint operators.

We conclude this section with a result which together with Proposition 4.1 characterizes those whos that are hyperoperators.

Proposition 4.6. Let $a=(a, D)$ be a who such that the closure of $\phi(a)$ is bounded on $X$ for all $\phi \in \mathcal{D}\left(\mathbb{R}^{n}\right)$. Assume that $\cap_{\phi \in \mathcal{D}} \operatorname{Ker} \overline{\phi(a)}=\{0\}$. Then the mapping $A$ defined by $A(\phi)=\overline{\phi(a)}$ is a hyperoperator with $D_{A}=\cup_{\phi \in \mathcal{D}} \operatorname{Im} \overline{\phi(a)} \supseteq D$. Moreover if $a^{\prime}$ is the who associated to $A$ then $\overline{a^{\prime}}=\bar{a}$.

Let $X$ and $a$ be as in Example 2, Then $(a, \mathcal{D}(\mathbb{R}))$ is a who satisfying the hypotheses of the proposition. The induced hyperoperator is $A=$ $[a]$ and $D_{A}$ is the space of all $f$ in $X$ with compact support.

Proof. We first show that $A$ so defined is a continuous mapping $\mathcal{D}\left(\mathbb{R}^{n}\right) \rightarrow$ $\mathrm{\ell}(X)$. To this end, we take a compact set $K \subset \mathbb{R}^{n}$, and a cutoff function $\chi$ that is 1 in a neighborhood of $K$. For each $x \in X$ we can define a mapping $A_{x}: \mathcal{E}\left(\mathbb{R}^{n}\right) \rightarrow X$ by $A_{x} f=\overline{(\chi f)(a)} x$. For $x \in D_{1}=D \cap\{|x| \leq 1\}$ the mapping $A_{x}$ is continuous, since $(a, D)$ is a who. By the Banach-Steinhaus theorem it follows that $\left\{A_{x}\right\}_{x \in D_{1}}$, $D_{1}=D \cap\{|x| \leq 1\}$, is equi-continuous, which means that

$$
\left|A_{x} f\right| \leq C \sum_{|\alpha| \leq M} \sup _{K^{\prime}}\left|\partial^{\alpha} f\right|
$$

for some $C, M$ and $K^{\prime}$ independent of $x \in D_{1}$. Applying to $\phi$ with support in $K$, and using that $D$ is dense, we get

$$
\|\overline{\phi(a)}\| \leq C \sum_{|\alpha| \leq M} \sup \left|\partial^{\alpha} \phi\right|
$$

Thus $A$ is continuous. The multiplicativity $A(\phi \psi)=A(\phi) A(\psi)$ now follows by continuity, since it holds when applied to $x \in D$. Moreover, for any $x \in D$ the map $\mathcal{E}\left(\mathbb{R}^{n}\right) \ni f \mapsto f(a) x \in X$ is continuous and therefore has compact support, $\sigma_{x}(a)$. If $\chi=1$ in a neighborhood of $\sigma_{x}(a)$ it follows that $\chi(a) x=1(a) x=x$. Hence $A$ is a hyperoperator with $D_{A}=\cup_{\phi \in \mathcal{D}} \operatorname{Im} \overline{\phi(a)} \supseteq D$.

It remains to see that $\overline{a^{\prime}}=\bar{a}$. If $\chi_{N}$ is an exhausting sequence, then $\psi_{N}=\xi \chi_{N} \rightarrow \xi$ in $\mathcal{E}\left(\mathbb{R}^{n}\right)$ and so for $x \in D \subseteq D_{A}$ we have

$$
a x=\lim _{N \rightarrow \infty} \psi_{N}(a) x=\lim _{N \rightarrow \infty} A\left(\psi_{N}\right) x=a^{\prime} x .
$$

Hence $a \subseteq a^{\prime}$ and so $\bar{a} \subseteq \overline{a^{\prime}}$. To obtain the converse inclusion it suffices to show that $\overline{\operatorname{Graph}(a)} \supseteq \operatorname{Graph}\left(a^{\prime}\right)$. Let $\left(x, a^{\prime} x\right) \in \operatorname{Graph}\left(a^{\prime}\right)$. Since $x \in D_{A}$, there is an $N_{0}$ such that $A\left(\chi_{N_{0}}\right) x=x$. Take any sequence $y_{j}$ 
in $D$ converging to $x$ and put $x_{j}=\chi_{N_{0}}(a) y_{j}$. Then $x_{j}$ is a sequence in $D$ and it also converges to $x$ since $\chi_{N_{0}}(a)$ has a bounded extension. It follows that

$$
a x_{j}=\lim _{N \rightarrow \infty} \psi_{N}(a) x_{j}=\lim _{N \rightarrow \infty} \psi_{N}(a) \chi_{N_{0}}(a) y_{j}=\psi_{N_{0}}(a) y_{j} \rightarrow \overline{\psi_{N_{0}}(a)} x,
$$

as $j \rightarrow \infty$. However, $\overline{\psi_{N_{0}}(a)} x=a^{\prime} x$ and hence $\left(x_{j}, a x_{j}\right) \rightarrow\left(x, a^{\prime} x\right)$, that is, $\left(x, a^{\prime} x\right) \in \overline{\operatorname{Graph}(a)}$.

Remark 2. Let $(a, D)$ be a who. For each $x \in D$ the mapping $\phi \mapsto$ $\phi(a) x$ is a continuous mapping $A_{x}: \mathcal{E}\left(\mathbb{R}^{n}\right) \rightarrow X$, and hence it has compact support. As for a hyperoperator, we can define the local spectrum $\sigma_{x}(a)$ as this support. If $D_{K}=\left\{x \in D ; \sigma_{x}(a) \subset K\right\}$, then clearly $D=\cup D_{K}$. For each $x \in D_{K}$ we have an estimate like (4.1), where $K^{\prime}$ is a compact neighborhood of $K$. However, in general this estimate cannot be uniform in $x$ for $|x| \leq 1$, since otherwise $\phi(a)$ would have a bounded extension to $X$.

To see how this lack of uniformity may appear, assume that $a=f(b)$ for some hyperoperator $b$, where $f$ takes values in $K \subset \subset \mathbb{R}^{m}$. Then $D_{K, a}=D_{b}=D_{a}$ because if $x \in D_{b}$ then $\chi(b) x=x$ and since $b$ has finite order on supp $\chi$ we get

$$
\begin{aligned}
|\phi(a) x|=|(\phi \circ f \cdot \chi)(b) x| & \leq C \sum_{|\alpha| \leq N_{\chi}} \sup \left|\partial^{\alpha}(\phi \circ f \cdot \chi)\right||x| \\
& \leq C \sum_{|\alpha+\beta+\gamma| \leq N_{\chi}} \sup \left|\partial^{\beta} f\right|\left|\partial^{\gamma} \chi\right| \sup _{K}\left|\partial^{\alpha} \phi\right||x| .
\end{aligned}
$$

However, $N_{\chi}$ and $\sup \left|\partial^{\beta} f \| \partial^{\gamma} \chi\right|$ may blow up as $\chi \rightarrow 1$.

\section{Spectrum of a hyperoperator}

We first recall

Proposition 5.1. Suppose that $a=\left(a_{1}, \ldots, a_{n}\right)$ is a tuple of bounded commuting operators with real spectra and resolvents with temperate growths, and $A$ is the corresponding hyperoperator on $\mathbb{R}^{n}$. Then $\operatorname{supp} A$ is equal to the (Taylor) spectrum of a.

For a proof, see [3]. In view of this result the following definition is natural.

Definition 3. For $A \in H_{\mathcal{D}\left(\mathbb{R}^{n}\right)}(X)$, the spectrum $\sigma(A)$ is the support of $A$ as a distribution.

When $A$ is identified with the who $a$ we often write $\sigma(a)$ instead of $\sigma(A)$. Notice that $A(\phi)$ only depends on the values of $\phi$ in a small neighborhood of $\sigma(A)$. If the spectrum of $A$ is compact, then clearly $A$ has a continuous extension to a multiplicative mapping $\mathcal{E}\left(\mathbb{R}^{n}\right) \rightarrow \mathrm{E}(X)$. For such an $A$ and $f \in \mathcal{E}\left(\mathbb{R}^{n}\right)$, we have that $f(a) x=\lim A\left(f \chi_{N}\right) x=$ 
$A(f) x$ for $x \in D$, and thus the closure of $f(a)$ is equal to the bounded operator $A(f)$. Applying to the identity mapping $\xi \mapsto \xi$ on $\mathbb{R}^{n}$ we get

Proposition 5.2. Suppose that $A \in H_{\mathcal{D}\left(\mathbb{R}^{n}\right)}(X)$ and $\sigma(A)$ is compact in $\mathbb{R}^{n}$. Then the closure $\bar{a}$ of a is bounded, and $[a]=A$. Moreover, $\sigma(A)$ coincides with the Taylor spectrum of $\bar{a}$.

If $f \in \mathcal{E}\left(\mathbb{R}^{n}\right)$ has its support in the complement of $\sigma(A)$, then $f(a) x=0$ for all $x \in D$, so the closure of $f(a)$ is $0_{X}$.

Definition 4. For a who $b=(b, D)$ we introduce the weak spectrum $\sigma_{w}(b)$ defined as the intersection of all closed sets $F$ such that $\phi(b) x=0$ for all $x \in D$ and $\phi$ with support in $\mathbb{R}^{n} \backslash F$.

Thus a point $p$ is outside $\sigma_{w}(b)$ if and only if for all $\phi$ with support sufficiently close to $p$ we have $\phi(b) x=0$ for all $x \in D$. It follows that if $b$ happens to be a hyperoperator then $\sigma_{w}(b)=\sigma(b)$. In particular, if $b x=0$ for all $x \in D$, then $\sigma_{w}(b)=\sigma(0)=\{0\}$.

Proposition 5.3. Let $b=(b, D)$ be a who and let $f \in \mathcal{E}\left(\mathbb{R}^{n}, \mathbb{R}^{m}\right)$. Then $\sigma_{w}(f(b))=f\left(\sigma_{w}(b)\right)$.

Proof. If $h \in \mathcal{D}\left(\mathbb{R}^{m}\right)$ has its support outside $f(\sigma(b))$, then $h \circ f$ vanishes in a neighborhood of $\sigma(b)$ so $(h \circ f)(b) x=0$ for $x \in D$, i.e, by definition, $h(f(b)) x=0$. This means that $\sigma_{w}(f(b)) \subset f\left(\sigma_{w}(b)\right)$. For the converse inclusion, take any point $p$ outside $\sigma_{w}(f(b))$ and let $h$ be a function identically equal to 1 in a neighborhood of $p$ and with support outside $\sigma_{w}(f(b))$. Then if $y \in f^{-1}(p)$ we have $h \circ f$ identically equal to 1 in a neighborhood of $y$. Hence for any $\phi$ with support in this neighborhood $\phi \cdot(h \circ f)=\phi$. Since $h$ has support outside $\sigma_{w}(f(b))$ we have $h \circ f(b) x=$ $h(f(b)) x=0$ for $x \in D$ and so $\phi(b) x=\phi \cdot(h \circ f)(b) x=\phi(b) h \circ f(b) x=0$ for $x \in D$. Thus $f^{-1}(p) \cap \sigma_{w}(b)=\emptyset$, i.e. $f\left(\sigma_{w}(b)\right) \subset \sigma_{w}(f(b))$.

Noting that $\sigma_{w}(b)=\sigma(b)$ when $b$ is a (strong) hyperoperator we immediately get

Corollary 5.4. If $A \in H_{\mathcal{D}\left(\mathbb{R}^{n}\right)}(X)$ and $f \in \mathcal{D}\left(\mathbb{R}^{n}, \mathbb{R}^{m}\right)$, or $f \in \mathcal{E}\left(\mathbb{R}^{n}, \mathbb{R}^{m}\right)$ is proper, then $\sigma(f(a))=f(\sigma(a))$.

Since $\sigma_{w}\left(0_{D_{A}}\right)=\sigma\left(0_{X}\right)=\{0\}$ we have

Corollary 5.5. If $A \in H_{\mathcal{D}\left(\mathbb{R}^{n}\right)}(X)$ and $f \in \mathcal{E}\left(\mathbb{R}^{n}, \mathbb{R}^{m}\right)$ and $f(a) x=0$ for all $x \in D_{A}$, then $\sigma(a) \subset f^{-1}(0)$.

It is not true in general that $f(\sigma(a))$ bounded implies that $f(a)$ is bounded (if $f$ is neither proper nor compactly supported). For instance, take $f(\xi)=\sin \xi^{m}$ and $a \sim \xi$ on $X=H^{1}(\mathbb{R})$. Then $|f| \leq 1$ on $\sigma(a)$ but $f(a)$, i.e., multiplication with $\sin \xi^{m}$ is not bounded on $X$. However we have

Lemma 5.6. If $a$ is a hyperoperator, $f \in \mathcal{E}\left(\mathbb{R}^{n}\right)$, and $b=f(a)$ is bounded, then $f(\sigma(a)) \subset \sigma(f(a))$. 
Proof. We know that $p \circ f(a)=p(b)$ for all polynomials. Let $\phi \in \mathcal{D}\left(\mathbb{R}^{n}\right)$ have support outside $\sigma(b)$ and take $p_{j}$ such that $p_{j} \rightarrow \phi$ in $\mathcal{E}\left(\mathbb{R}^{n}\right)$. Then $p_{j} \rightarrow 0$ uniformly in a neighborhood of $\sigma(b)$; we may even assume that this holds in a complex neighborhood; thus we can conclude that $p_{j}(b) \rightarrow 0$ (even though we do not know whether $b$ admits a smooth functional calculus or not!).

Moreover, $p_{j} \circ f \rightarrow \phi \circ f$ in $\mathcal{E}\left(\mathbb{R}^{n}\right)$ so $p_{j} \circ f(a) x \rightarrow \phi \circ f(a) x$ for $x \in D$. Since $p_{j} \circ f(a)=p_{j}(b) \rightarrow 0$ we conclude that $\phi \circ f(a)=0$. From Corollary [5.5 we get $f(\sigma(a)) \subset\{\phi=0\}$ and we conclude that $f(\sigma(a)) \subset \sigma(b)$.

Proposition 5.7. Assume that $a=(a, D)$ is a who and that the closure of $r_{z}(a)$ is bounded for each $r_{z}(\xi), z \in \mathbb{C} \backslash \mathbb{R}$. Then $\bar{a}$ has real spectrum in the usual sense.

Proof. We first prove that the closure $b$ of $r(a)=r_{i}(a)$ is the inverse of $\bar{a}+i$. We know that $(a+i) b x=x=b(a+i) x$ for $x \in D$. Suppose that $x \in \operatorname{Dom}(\bar{a}+i)=\operatorname{Dom}(\bar{a})$. Then there are $x_{j} \in D$ such that $x_{j} \rightarrow x$ and $(a+i) x_{j} \rightarrow(\bar{a}+i) x$. Since $b$ is bounded we have

$$
x \leftarrow x_{j}=b(a+i) x_{j} \rightarrow b(\bar{a}+i) x
$$

so $b(\bar{a}+i) x=x$ for $x \in \operatorname{Dom}(\bar{a}+i)$. Moreover, if $x$ is arbitrary and $x_{j} \in D$ and $x_{j} \rightarrow x$, then $b x_{j} \rightarrow b x$ and $(a+i) b x_{j}=x_{j} \rightarrow x$ so by definition $b x$ is in the domain of $\bar{a}+i$ and $(\bar{a}+i) b x=x$.

\section{Representation By PSEUdORESOLVENTS}

We first consider the case $n=1$. If $a$ is an HS operator, then we have the representation (1.2) of $\phi(a)$. For a general $a \in H_{\mathcal{D}(\mathbb{R})}(X)$ such a representation cannot hold simply because the resolvent is not defined. We will discuss various ways to obtain formulas that will replace (1.2). The simplest way is to use cut-off functions $\chi$ and define

$$
\omega_{\zeta-a}^{\chi}=\left.\chi(\xi) \omega_{\zeta-\xi}\right|_{\xi=a}, \quad \omega_{\zeta-\xi}=d \zeta /(\zeta-\xi) 2 \pi i .
$$

Proposition 6.1. Suppose that $a \in H_{\mathcal{D}(\mathbb{R})}(X)$. Then $\omega_{\zeta-a}^{\chi}$ is holomorphic for $|\operatorname{Im} \zeta|>0$ and

$$
\left\|\omega_{\zeta-a}^{\chi}\right\|=\varnothing\left(|\operatorname{Im} \zeta|^{-M}\right)
$$

for some $M$. If $\phi \in \mathcal{D}(\mathbb{R})$ and $\operatorname{supp} \phi \subset \subset\{\chi=1\}$, then

$$
\phi(a)=\int \omega_{\zeta-a}^{\chi} \wedge \bar{\partial} \tilde{\phi}(\zeta)
$$

Proof. By Lemma $2.1 \omega_{z-a}^{\chi}$ is strongly holomorphic in $\mathbb{C} \backslash \mathbb{R}$. Since $A$ has finite order on $K \supset \supset \operatorname{supp} \chi, A(\psi)$ only depends on a finite number of derivatives of $\psi$ if $\operatorname{supp} \psi \subset K$ and so we get (6.11). If

$$
\phi_{\epsilon}(\xi)=\frac{1}{2 \pi i} \int_{|\operatorname{Im} \zeta|>\epsilon} \frac{\chi(\xi) d \zeta}{\zeta-\xi} \wedge \bar{\partial} \tilde{\phi}(\zeta)
$$


it is readily checked, for instance by approximating by Riemann sums, that

$$
\phi_{\epsilon}(a)=\int_{|\operatorname{Im} \zeta|>\epsilon} \omega_{\zeta-a}^{\chi} \wedge \bar{\partial} \tilde{\phi}(\zeta) .
$$

Moreover, $\phi_{\epsilon} \rightarrow \phi \chi=\phi$ in $\mathcal{D}(\mathbb{R})$, and hence $\phi_{\epsilon}(a) \rightarrow \phi(a)$. Because of (6.1) it follows that the right hand side of (6.2) is absolutely convergent and equal to the limit of the right hand side of (6.3).

Proposition 6.2. Each $\omega_{z-a}^{\chi}$ has a holomorphic continuation to the set $\mathbb{C} \backslash \sigma(a)$; more precisely, $\mathbb{C} \backslash \sigma(a)$ is precisely the set where all $\omega_{\zeta-a}^{\chi}$ are strongly holomorphic.

Proof. The first statement is proved analogously to Lemma 2.1. If $x \in$ $\mathbb{R} \backslash \sigma(a)$, let $\tilde{\chi}$ be a cut-off function that is equal to $\chi$ in a neighborhood of $\sigma(a)$ and zero in a neighborhood of $x$. Then $\omega_{z-a}^{\chi}=A(\tilde{\chi} /(z-\xi))$ and imitating the proof of Lemma 2.1] we see that $\omega_{z-a}^{\chi}$ is strongly holomorphic close to $x$. For the converse, assume $\phi$ has its support where $\omega_{\zeta-a}^{\chi}$ is holomorphic and $\chi$ identically 1 in a neighborhood of $\operatorname{supp} \phi$. Then by Proposition 6.1.

$$
A(\phi)=\int \omega_{z-a}^{\chi} \wedge \bar{\partial} \tilde{\phi}(z)=-\int \bar{\partial}\left(\tilde{\phi}(z) \omega_{z-a}^{\chi}\right)=0
$$

by Stokes' theorem and thus we are done.

The advantage with the usual representation (1.2) is of course that a priori we only have to compute $\phi(a)$ for $\phi(\xi)=1 /(\zeta-\xi)$. For the general hyperoperator we must insert various functions $\chi$ as well. However, if we impose growth restrictions on $[a]$, one single formula will do. In Section 7 we will consider the case with polynomial growth restrictions.

If $a$ is a hyperoperator or even just a who, then for each $x \in D$, the resolvent $\omega_{\zeta-a} x$ is holomorphic outside the compact set $\sigma_{x}(a) \subset \mathbb{R}$, and from (4.1) we have that $\left|\omega_{\zeta-a} x\right| \leq C|\operatorname{Im} \zeta|^{-M}$. With a similar argument as above we therefore have the representation

$$
\phi(a) x=\int \omega_{\zeta-a} x \wedge \bar{\partial} \tilde{\phi}(\zeta), \quad \phi \in \mathcal{E}(\mathbb{R}) .
$$

Recall that $\mathcal{G}(\mathbb{R})$ is the algebra of functions on $\widehat{\mathbb{R}}$ that are holomorphic in a complex neighborhood of $\infty$. Convergence in $\mathcal{G}(\mathbb{R})$ of a sequence $f_{j}$ means that $f_{j}$ converges in $\mathcal{E}(\widehat{\mathbb{R}})$ and moreover, that all $f_{j}$ are holomorphic in a fixed complex neighborhood of $\infty$ and converge uniformly on compacts in this neighborhood.

Theorem 6.3. A hyperoperator $A \in H_{\mathcal{D}(\mathbb{R})}(X)$ corresponds to an $H S$ operator if and only if $A: \mathcal{D}(\mathbb{R}) \rightarrow E(X)$ has a multiplicative continuous extension to a mapping $\mathcal{G}(\mathbb{R}) \rightarrow E(X)$. 
This result was more or less proved in [3]; one part is contained in the proof of Proposition 7.2 in [3] and the other part is stated in Proposition 11.4 in the same paper, but for the reader's convenience we supply a proof here.

Proof. First we notice that such an extension of $A$ must be unique if it exists at all. In fact, for any $\psi \in \mathcal{G}(\mathbb{R})$ and $x \in D_{A}$ we have $A(\psi \chi) x=$ $\psi(a) x$ if $\chi$ is chosen so that $A(\chi) x=x$. On the other hand if $\hat{A}$ is a multiplicative extension of $A$ we get $\hat{A}(\psi) x=\hat{A}(\psi) A(\chi) x=A(\psi \chi) x$ Hence $\hat{A}(\psi)$ coincides with $\psi(a)$ on $D_{A}$ and since $D_{A}$ is dense and $\hat{A}(\psi)$ is bounded this uniquely determines $\hat{A}(\psi)$. Here $a$ denotes the who associated to $A$.

For the "only if"-part we first assume that (the closure of) $a$ is an HS operator, cf., Proposition 4.5. Then the action of $A$ is given by (1.2) and we want to extend this formula to any function $f$ in $\mathcal{G}(\mathbb{R})$. Let $F$ be the holomorphic extension to a complex neighborhood $O$ of $\infty$, and let $\chi$ be a cut-off function in $\mathbb{R}$ that is equal to 1 in a neighborhood of $K=\mathbb{R} \backslash(\mathbb{R} \cap O)$. One can find an almost holomorphic extension $\tilde{\chi}$ which is 0 in a complex neighborhood of $\infty$ and 1 in a complex neighborhood of $K$. Then $\tilde{f}=(1-\tilde{\chi}) F+\widetilde{\chi f}$ is an almost holomorphic extension of $f$ to a complex neighborhood of $\widehat{\mathbb{R}}$ in $\widehat{\mathbb{C}}$ which is holomorphic in a neighborhood of $\infty$. Let $\psi$ be a function identically equal to 1 in a neighborhood of $\widehat{\mathbb{R}}$ in $\widehat{\mathbb{C}}$ and with support in a slightly larger neighborhood avoiding the point $i$. Then

$$
\frac{1}{2 \pi i} \int \frac{a-i}{\zeta-i} \frac{d \zeta}{\zeta-a} \wedge \bar{\partial}(\tilde{f} \psi(\zeta))
$$

provides the desired extension. In fact, if $f$ has compact support then $\tilde{f} \psi$ is an almost holomorphic extension of $f$ with compact support avoiding $i$. It follows by Stokes' theorem that formula (6.4) yields the same operator as (1.2). Moreover (6.4) is continuous and multiplicative on $\mathcal{G}(\mathbb{R})$. This is perhaps most easily seen by pulling back to the unit circle $\mathbb{T}$. The Cayley transform $b=C(a)$, cf., (2.1), is a bounded operator with spectrum contained in the unit circle $\mathbb{T}$, and

$$
\frac{1}{2 \pi i} \int \frac{a-i}{\zeta-i} \frac{d \zeta}{\zeta-a} \wedge \bar{\partial}(\tilde{f} \psi(\zeta))=\frac{1}{2 \pi i} \int \frac{d w}{w-b} \wedge \bar{\partial}\left(\tilde{f} \psi\left(C^{-1}(w)\right)\right) .
$$

The right hand side is a continuous extension of the holomorphic functional calculus for $b$ to the space of smooth functions on $\mathbb{T}$ which are analytic in a neighborhood of 1 since $\left\|(w-b)^{-1}\right\|$ has tempered growth in $\mathbb{T} \backslash\{1\}$. Since the analytic functions are dense in this space, the multiplicativity follows automatically.

Conversely, assuming that $A$ is a hyperoperator that admits an extension to $\mathcal{G}(\mathbb{R})$, we want to prove that $\bar{a}$ is an HS operator. Since $A$ now operates on all $r_{z}(\xi)=1 /(z-\xi)$ it follows from Proposition 5.7 that $\bar{a}$ has spectrum in $\mathbb{R}$ in the usual sense. Clearly then $r_{z}(a) d z / 2 \pi i$ 
is the resolvent of $\bar{a}$. Given a compact $K \subset \mathbb{R}$ take $\chi$ and $\tilde{\chi}$ as above. As $A$ has finite order $m$ on $K^{\prime}=\operatorname{supp} \chi$ it follows that

$$
\left\|\frac{\chi(a)}{z-a}\right\| \leq C_{K}|\operatorname{Im} z|^{-(m+1)}
$$

for any $z \in \mathbb{C} \backslash \mathbb{R}$. For $z$ in a small neighborhood of $K$, the functions

$$
g_{z}(\xi)=\frac{\tilde{\chi}(z)-\chi(\xi)}{z-\xi} .
$$

are uniformly bounded in $\mathcal{G}(\mathbb{R})$, and by (6.5) so are $\left\|g_{z}(a)\right\|=\left\|A\left(g_{z}\right)\right\|$. Thus (1.1) follows by the triangle inequality.

Remark 3. Let $a \in H_{\mathcal{D}(\mathbb{C})}(X)$. Then we can define $\omega_{\zeta-a}^{\chi}$ as a $\mathrm{E}(X)$ valued distribution $((1,0)$-current) in $\mathbb{C}$ by

$$
\omega_{\zeta-a}^{\chi} \cdot \psi d \bar{\zeta}=\left.\frac{1}{2 \pi i} \int_{\zeta} \frac{\chi(\xi) \psi(\zeta) d \zeta \wedge d \bar{\zeta}}{\zeta-\xi}\right|_{\xi=a}, \quad \psi \in \mathcal{D}(\mathbb{C})
$$

If we apply to $\bar{\partial} \psi$ we get $\omega_{\zeta-a}^{\chi} . \bar{\partial} \psi=\left.\chi(\xi) \psi(\xi)\right|_{\xi=a}=\chi(a) \psi(a)$. Thus $\bar{\partial} \omega_{\zeta-a}^{\chi}=\chi[a]$. If in fact $a \in H_{\mathcal{D}(\mathbb{R})}(X)$ and we choose $\psi=\tilde{\phi}$ in (6.6), then we can move $a$ inside the integral and thus get back (6.2). However, in general it is not possible to put $a$ inside the integral.

If we want an absolutely convergent integral representation for $\phi(a)$ when $a \in H_{\mathcal{D}\left(\mathbb{R}^{n}\right)}(X)$ we can use the Bochner-Martinelli form

$$
\omega_{\xi}=b(\xi) \wedge(\bar{\partial} b(\xi))^{n-1}, \quad b(\xi)=\frac{\sum \bar{\xi}_{j} d \xi_{j}}{2 \pi i|\xi|^{2}}
$$

and define $\omega_{\zeta-a}^{\chi}=\left.\chi(\xi) \omega_{\zeta-\xi}\right|_{\xi=a}$. Then $\omega_{\zeta-a}$ is $\bar{\partial}$-closed in $\mathbb{C}^{n} \backslash \mathbb{R}^{n}$ and the analogue of Proposition 6.1 holds. Proposition 6.2 also has a generalization to the $\mathbb{R}^{n}$ case; $\mathbb{C}^{n} \backslash \sigma(a)$ is precisely the set where $\omega_{z-a}^{\chi}$ is strongly $\bar{\partial}$-closed. If we consider a hyperoperator $a \in H_{\mathcal{D}\left(\mathbb{R}^{2 n}\right)}(X)$ as an element in $a \in H_{\mathcal{D}\left(\mathbb{C}^{n}\right)}(X)$, the analog of Remark 3 also holds.

Tensor products of hyperoperators can also be defined by integral formulas. Assume that $A_{1}, \ldots, A_{m}$ are in $H_{\mathcal{D}\left(\mathbb{R}^{\left.n_{j}\right)}\right.}$ but not necessarily commuting. Then we can form the tensor product $A=A_{1} \otimes \cdots \otimes A_{m}$, and obtain a linear continuous, though not multiplicative, operator $\mathcal{D}\left(\mathbb{R}^{n}\right) \rightarrow \mathrm{E}(X)$, where $n=n_{1}+\cdots+n_{m}$. For $\phi \in \mathcal{D}\left(\mathbb{R}^{n}\right)$ we can find an almost holomorphic extension $\tilde{\phi}$ such that (2.5) holds. In [3] this is only proved when all $n_{j}=1$ but the general case follows along the same lines. Then

$$
\left(A_{1} \otimes \cdots \otimes A_{m}\right)(\phi)=\int \omega_{\zeta_{1}-a_{1}}^{\chi_{1}} \wedge \ldots \wedge \omega_{\zeta_{m}-a_{m}}^{\chi_{m}} \wedge \bar{\partial}_{\zeta_{m}} \cdots \bar{\partial}_{\zeta_{1}} \tilde{\phi}(\zeta)
$$

if the support of $\phi$ is contained in the set where $\chi_{1} \otimes \cdots \otimes \chi_{m}=1$. To see this, first notice that the integral makes sense in view of the assumption (2.5) and the estimates (6.1) of $\omega_{\zeta_{j}-a_{j}}^{\chi_{j}}$. Since (6.7) clearly holds for $\phi$ of the form $\phi=\phi_{1} \otimes \cdots \otimes \phi_{m}$, the general case follows by 
continuity. One can also prove directly that (6.7) is independent of the choice of special almost analytic extension $\tilde{\phi}$ along the lines in [3, and then use this as the definition of the tensor product.

Remark 4. We can also generalize Theorem 6.3 to several variables, and we illustrate by considering a hyperoperator $A \in H_{\mathcal{D}\left(\mathbb{R}^{2}\right)}(X)$. First we define $\mathcal{G}\left(\mathbb{R}^{2}\right)$ as the union (direct limit) of the spaces $\mathcal{G}_{U}\left(\mathbb{R}^{2}\right), U$ a complex neighborhood of $\infty$ in $\widehat{\mathbb{C}}$, defined as all smooth functions $f$ on $\widehat{\mathbb{R}} \times \widehat{\mathbb{R}}$ which are holomorphic on $U \times U$ and such that $x \mapsto f(x, y)$ is holomorphic in $U$ for any $y$ and $y \mapsto f(x, y)$ is holomorphic in $U$ for any $x$. A sequence $f_{j}$ in $\mathcal{G}\left(\mathbb{R}^{2}\right)$ converges if all $f_{j}$ are in some fixed $\mathcal{G}_{U}\left(\mathbb{R}^{2}\right)$ and converges in $\mathcal{E}((\widehat{\mathbb{R}} \cup U) \times(\widehat{\mathbb{R}} \cup U))$. The analog of Theorem 6.3 is: $A$ has a continuous extension to $\mathcal{G}\left(\mathbb{R}^{2}\right)$ if and only if the closures of $a_{j}=A\left(\pi_{j}\right), j=1,2$, are of HS type and commute strongly, i.e., their resolvents commute. Notice however that this condition highly depends on the choice of coordinates on $\mathbb{R}^{2}$, whereas the notion of general hyperoperator is coordinate invariant.

\section{TEMPERATE HYPEROPERATORS}

We say that $A \in H_{\mathcal{D}\left(\mathbb{R}^{n}\right)}(X)$ is temperate, $A \in H_{\mathcal{S}\left(\mathbb{R}^{n}\right)}(X)$, if it extends to a (necessarily multiplicative) mapping $\mathcal{S}\left(\mathbb{R}^{n}\right) \rightarrow \mathrm{E}(X)$.

Since $\mathcal{D}\left(\mathbb{R}^{n}\right)$ is dense in $\mathcal{S}\left(\mathbb{R}^{n}\right)$ it follows that a continuous multiplicative map $\mathcal{S}\left(\mathbb{R}^{n}\right) \rightarrow \mathrm{E}(X)$ satisfies $(i)$ and $(i i)$ in Definition 1 if and only if it holds with $\mathcal{D}\left(\mathbb{R}^{n}\right)$ replaced by $\mathcal{S}\left(\mathbb{R}^{n}\right)$ (but the corresponding dense domain may be larger).

For standard functional analysis reasons it follows that for any temperate $A$ there is an integer $M$ such that

$$
|A(\phi)| \leq C \sum_{|\alpha|,|\beta| \leq M} \sup _{\mathbb{R}^{n}}\left|\xi^{\beta} \partial^{\alpha} \phi\right|,
$$

which in particular means that $A(\phi)$ is defined for $\phi$ such that its derivatives up to order $M$ as least have decay like $1 /|\xi|^{M}$.

Example 5. Let $X$ be the set of functions $\phi(\xi)$ on $\mathbb{R}$ with norm $\|\phi\|=$ $\sum_{\ell}\|\phi\|_{C^{\ell}\left(K_{\ell+1} \backslash \operatorname{int} K_{\ell-1}\right)}$. Then multiplication with $\xi\left(2+\sin \xi^{3}\right)$ is a hyperoperator that is not temperate.

The multiplication hyperoperator $f(\xi)=\xi\left(2+\sin \xi^{3}\right)$ on $H^{1}(\mathbb{R})$ from Example 2 is a tempered hyperoperator, which has no ordinary resolvent. Notice, though, that

$$
\frac{i+\zeta}{i+f(\xi)} \frac{1}{\zeta-f(\xi)}
$$


is bounded for all $\zeta \in \mathbb{C} \backslash \mathbb{R}$. More generally, if $A \in H_{\mathcal{S}(\mathbb{R})}(X)$ and $m$ is a large enough integer we can define, in view of (7.1),

$$
\omega_{\zeta-a}^{m}=\left.\left(\frac{i+\zeta}{i+\xi}\right)^{m} \omega_{\zeta-\xi}\right|_{\xi=a},
$$

for $\zeta \in \mathbb{C} \backslash \mathbb{R}$. If $A \in H_{\mathcal{S}\left(\mathbb{R}^{n}\right)}(X)$ we can take instead

$$
\omega_{\zeta-a}^{m}=\left.\left(\frac{1+\zeta \cdot \xi}{1+|\xi|^{2}}\right)^{m} \omega_{\zeta-\xi}\right|_{\xi=a} .
$$

for $\zeta \in \mathbb{C}^{n} \backslash \mathbb{R}^{n}$.

Proposition 7.1. The form $\omega_{\zeta-a}^{m}$ is $\bar{\partial}$-closed in $\mathbb{C}^{n} \backslash \mathbb{R}^{n}$ and admits a $\bar{\partial}$-closed extension to $\mathbb{C} \backslash \sigma(a)$. Moreover, if $\phi \in \mathcal{S}\left(\mathbb{R}^{n}\right)$ and $\tilde{\phi}$ is an appropriate almost holomorphic extension, then

$$
A(\phi)=\int \omega_{\zeta-a}^{m} \wedge \bar{\partial} \tilde{\phi} .
$$

This means, cf., Remark 3, that $\bar{\partial} \omega_{\zeta-a}^{m}=[a]$. Moreover, if $\omega_{\zeta-a}^{m}$ has a $\bar{\partial}$-closed extension to $\mathbb{C}^{n} \backslash F$, then $\sigma(a) \subset F$.

Sketch of proof. First notice that

$$
\sup _{\xi \in \mathbb{R}^{n}}\left|\xi^{\beta} \partial_{\xi}^{\alpha} \omega_{\zeta-\xi}^{m}\right| \leq C \frac{(1+|\zeta|)^{m}}{|\operatorname{Im} \zeta|^{2 n-1+|\alpha|}}
$$

if just $|\beta|<m$. If $A$ satisfies (7.1), therefore $\omega_{\zeta-a}^{m}$ is well-defined if $m \geq M$, and

$$
\left\|\omega_{\zeta-a}^{m}\right\| \leq C \frac{(1+|\zeta|)^{m}}{|\operatorname{Im} \zeta|^{2 n-1+|\alpha|}} .
$$

Given $\phi \in \mathcal{S}\left(\mathbb{R}^{n}\right)$ we let

$$
\tilde{\phi}(\zeta)=\int_{t} e^{i t \cdot \zeta} \hat{\phi}(t) \chi\left(\sqrt{1+|t|^{2}}|\operatorname{Im} \zeta|\right),
$$

where $\chi(s)$ smooth, supported in the unit ball in $\mathbb{R}^{n}$ and identically 1 in a neighborhood of the origin. One easily checks that $\tilde{\phi}(\zeta)$ is smooth, and equal to $\phi$ on $\mathbb{R}^{n}$, and that moreover,

$$
\bar{\partial} \tilde{\phi}(\zeta)=\varnothing_{M_{1}, M_{2}}\left(|\operatorname{Im} \zeta|^{M_{1}}(1+|\zeta|)^{-M_{2}}\right), \quad M_{1}, M_{2}>0
$$

In view of (7.3), therefore, the integral in (7.2) is well-defined. Moreover, from (17.4) it is easily seen that $\int \omega_{\zeta-\xi} \wedge \bar{\partial} \tilde{\phi}(\zeta)=\phi(\xi)$, and replacing $\tilde{\phi}(\zeta)$ by

$$
\tilde{\phi}(\zeta)\left(\frac{1+\zeta \cdot \xi}{1+|\xi|^{2}}\right)^{m}
$$

which satisfies a similar estimate, we get that

$$
\int \omega_{\zeta-\xi}^{m} \wedge \bar{\partial} \tilde{\phi}(\zeta)=\phi(\xi)
$$

One then proves (7.2) along the same lines as Proposition 6.1. 
For tempered hyperoperators the theory for tempered distributions is at our disposal. We will use this to prove a new form of Stone's theorem. We first recall a simple known variant.

Example 6. If $v \in C^{1}\left(\mathbb{R}^{n}, \mathrm{E}(X)\right)$ and

$$
v(t+s)=v(t) v(s), \quad v(0)=e_{X},
$$

then $v(t)=e^{i a \cdot t}$, for the commuting tuple $a_{k}=\left(\partial v / \partial t_{k}\right)(0) / i$ in $\mathrm{E}(X)$. If in addition $|v(t)|=\varnothing\left(|t|^{m}\right)$, when $|t| \rightarrow \infty$, then $\sigma(a) \subset \mathbb{R}^{n}$. If we only assume that $v(t)$ is continuous and satisfies (7.5), then the conclusion is not true. (For instance, if $n=1$ and $a$ is multiplication with $\xi$ on $L^{2}\left(\mathbb{R}_{\xi}\right)$, then $v(t)=e^{i a t}$ is multiplication by $e^{i \xi t}$ and thus a bounded operator, but $v^{\prime}(0)$ is not bounded.) However, $v$ is generated by a hyperoperator $A \in H_{\mathcal{S}\left(\mathbb{R}^{n}\right)}(X)$, i.e., $v(t)=\exp i a \cdot t$.

In fact, assume that $v(t)$ is continuous in the weak sense that $v(t) x$ is continuous for each $x \in X$. It then follows from the Banach-Steinhaus theorem that $\|v(t)\|$ is uniformly bounded on compact sets. Therefore, $v \cdot \phi=\int_{t} v(t) \phi(t) d t$ is a bounded operator for each $\phi \in \mathcal{S}\left(\mathbb{R}^{n}\right)$. Moreover, the condition $v(0)=e_{X}$ implies that $\cap \operatorname{Ker} v(\phi)=\{0\}$ and $\cup \operatorname{Im} v(\phi)$ is dense. In fact, let $\phi_{j} \rightarrow \delta_{0}$. Then $v\left(\phi_{j}\right) x \rightarrow x$ since $\varphi \mapsto v(\varphi) x$ is continuous and we easily see that $\cap \operatorname{Ker} v(\phi)=\{0\}$ and that $\cup \operatorname{Im} v(\phi)$ is dense. The existence of the generator $A$ now follows from Proposition 7.2 below.

Let $A$ be a tempered hyperoperator and let $D=\cup_{\phi \in \mathcal{S}\left(\mathbb{R}^{n}\right)} \operatorname{Im} A(\phi)$. If $f \in \mathcal{E}\left(\mathbb{R}^{n}\right)$ is a multiplier on $\mathcal{S}\left(\mathbb{R}^{n}\right)$, i.e., $f \mathcal{S}\left(\mathbb{R}^{n}\right) \subset \mathcal{S}\left(\mathbb{R}^{n}\right)$, we can define $f(a) x$ for $x \in D$ as $A(f \phi) y$ if $x=A(\phi) y$. To see that this is well-defined, assume that also $x=A\left(\phi^{\prime}\right) y^{\prime}$. By the multiplicativity, we then have that $A\left(\chi_{N} f \phi\right) y=A\left(\chi_{N} f \phi^{\prime}\right) y^{\prime}$ since $\chi_{N} f$ is in $\mathcal{S}$. When $N \rightarrow \infty, \chi_{N} f \phi \rightarrow f \phi$ in $\mathcal{S}$, and hence $A(f \phi) y=A\left(f \phi^{\prime}\right) y^{\prime}$. It is readily checked that $f(a)$ maps $D \rightarrow D$ and that $(f g)(a) x=f(a) g(a) x$.

Observe that $f(\xi)=\exp (i \xi \cdot t)$ is a multiplier on $\mathcal{S}$, so $\exp (i a \cdot t) x$ is defined for all $x \in D$. Moreover, $x=A(\phi) y$ so $\exp (i a \cdot t) x=$ $A(\phi(\xi) \exp (i \xi \cdot t)) y$, and therefore (17.1) implies that

$$
\left|e^{i a \cdot t} x\right| \leq C_{x}|t|^{M} \text {. }
$$

We claim that

$$
A(\hat{\psi}) x=\int_{t} \psi(t) e^{-i a \cdot t} x d t, \quad \psi \in \mathcal{S}, x \in D .
$$

In fact, the integral is convergent in view of (77.6) and it is easy to see that it is equal to $A(\hat{\psi}) x$ since $\int_{|t|<R} \psi(t) e^{-i \xi \cdot t} d t \rightarrow \hat{\psi}(\xi)$ in $\mathcal{S}$. In particular, the integral in (7.7) has a continuous extension to $X$. Since $A$ is in $\mathcal{S}^{\prime}$ it has a Fourier transform $\hat{A}$, defined by $\hat{A}(\psi)=A(\hat{\psi})$, and thus we have the suggestive formula $\hat{A}(t)=\exp (-i a \cdot t)$. If we let $v(t)=\exp (-i a \cdot t)=\hat{A}(t)$ then clearly $v(t+s) x=v(t) v(s) x$ for $x \in D$. 
Moreover, clearly $v$ defined by $v \cdot \psi=\hat{\psi}(a)$ satisfies

$$
\int_{s} \int_{t} v(t+s) \phi(t) \psi(s)=\int_{t} v(t) \phi(t) \int_{s} v(s) \psi(s), \quad \phi, \psi \in \mathcal{S}
$$

and

$$
\cap \operatorname{Ker} v(\phi)=\{0\}, \quad \cup \operatorname{Im} v(\phi)=D \text { dense. }
$$

We have the following variant of Stone's theorem.

Proposition 7.2. Assume that $v: \mathcal{S}\left(\mathbb{R}^{n}\right) \rightarrow E(X)$ is linear, and continuous in the sense that for fixed $x \in X, v . \phi_{j} x \rightarrow 0$ whenever $\phi_{j} \rightarrow 0$ in $\mathcal{S}\left(\mathbb{R}^{n}\right)$. Moreover, assume that $v(t)$ is group of operators in the sense of (7.8) and $v(0)=e_{X}$ in the sense of (7.9). Then $v$ is generated by a hyperoperator $A \in H_{\mathcal{S}\left(\mathbb{R}^{n}\right)}(X)$ in the sense that $v(t) x$ is smooth for $x \in D_{A}$ and $\left(\partial v / \partial t_{k}\right)(0) x=i a_{k} x$.

Proof. Define $A(\phi)=v \cdot \hat{\phi}$. By the Banach-Steinhaus theorem the pointwise continuity of $v$ implies strong continuity and so $A$ is a continuous map $\mathcal{S}\left(\mathbb{R}^{n}\right) \rightarrow \mathcal{L}(X)$. Moreover, the weak multiplicativity of $v$ implies that $v(\phi * \psi)=v(\phi) v(\psi)$ and hence

$$
A(\phi \psi)=v(\widehat{\phi \psi})=v(\hat{\phi} * \hat{\psi})=v(\hat{\phi}) v(\hat{\psi})=A(\phi) A(\psi) .
$$

Since the Fourier transform is an isomorphism of $\mathcal{S}\left(\mathbb{R}^{n}\right)$ we get that $\cap \operatorname{Ker} A(\phi)=\{0\}$ and $\cup \operatorname{Im} A(\phi)=D$ is dense. Thus $A$ is a tempered hyperoperator. For $x \in D$ we can define $u(t) x=e^{i a t} x$ and since $A$ satisfies an estimate like (7.1) it is easy to see that $|u(t) x| \leq C|t|^{M}$ and so $u(t) x$ defines an element in $\mathcal{S}^{\prime}\left(\mathbb{R}^{n}, X\right)$. We also see that $t \mapsto$ $u(t) x$ is in $C^{1}$ (even in $C^{\infty}$ ) and $u^{\prime}(t) x=i a x$. In fact, if $\phi \in \mathcal{S}$ then $\phi(\xi)\left(e^{i \xi t}-1\right) / t \rightarrow i \xi \phi(\xi)$ in $\mathcal{S}$ as $t \rightarrow 0$, and hence if $x=A(\phi) y$ we get

$$
\frac{e^{i a t}-e_{X}}{t} x=\frac{\phi(a) e^{i a t}-\phi(a)}{t} y \rightarrow i a \phi(a) y=i a x .
$$

We finally check that $u(t) x=v(t) x$ as tempered distributions. If, as before, $x=A(\phi) y$, then for any $\psi \in \mathcal{S}$ we have

$$
\begin{aligned}
\int_{t} \psi(t) u(t) x & =\int_{t} \psi(t) A_{\xi}\left(\phi(\xi) e^{i \xi t}\right) y=A_{\xi}\left(\phi(\xi) \int_{t} \psi(t) e^{i \xi t}\right) y \\
& =A_{\xi}(\phi(\xi) \hat{\psi}(\xi)) y=A(\hat{\psi}) x=\int_{t} \psi(t) v(t) x
\end{aligned}
$$

\section{Operators With UltradifFerentiable FunCtional CALCULUS}

Let $h(t)=H(|t|)$ where $H(0)=0$ and $H$ increasing and concave on $[0, \infty)$. Then $h$ is subadditive. We also assume that $\lim _{|t| \rightarrow \infty} h(t) /|t|=$ 
0 and that

$$
\limsup _{|t| \rightarrow \infty} \frac{\log (1+|t|)}{h(t)}=0 .
$$

Let $\mathcal{A}_{h}$ be the space of tempered distributions $f$ on $\mathbb{R}^{n}$ such that $\hat{f}$ is a measure and

$$
\|f\|_{\mathcal{A}_{h}}=\int_{t}|\hat{f}(t)| e^{h(t)} d t<\infty
$$

Because of (8.1), $\mathcal{A}_{h}$ is contained in $C^{\infty}\left(\mathbb{R}^{n}\right)$. Clearly $\mathcal{A}_{h}$ is a Banach space of functions that is closed under translations, and since $h$ is subadditive it follows, see e.g., [2, that $\mathcal{A}_{h}$ actually is a Banach algebra under pointwise multiplication. These algebras were introduced by Beurling, [4]. If $h(t)=|t|^{\alpha}, 0<\alpha<1$, then $G_{\alpha}=\cup_{c>0} \mathcal{A}_{c h}$ is the classical Gevrey algebra, see [11]. We say that the class $\mathcal{A}_{h}$ is nonquasianalytic if for each compact set $E$ and open neighborhood $U \supset E$ there is a function $\chi \in \mathcal{A}_{h}$ with support in $U$ which is identically 1 in some neighborhood of $E$. We recall the following version of the Denjoy-Carleman theorem.

Theorem 8.1. The class $\mathcal{A}_{h}$ is non-quasianalytic if and only if

$$
\int_{1}^{\infty} \frac{H(s) d s}{s^{2}}<\infty
$$

Assume now that $h(t)=H(|t|)$ satisfies the condition (8.3). Let $B_{h}$ be the algebra of all functions on $\mathbb{R}^{n}$ which are locally in $\mathcal{A}_{c h}$ for some $c>1$, and let $B_{h, 0}$ be the subalgebra of functions with compact support. There is an associated convex decreasing function $G(s)=$ $\sup _{t}(H(t)-t s)$ on $(0, \infty)$. Let $H_{c}(s)=H(c s)$ and let $G_{c}$ be the corresponding decreasing function.

Proposition 8.2. A function $\phi \in B_{h}$ if and only if it admits an almost holomorphic extension $\tilde{\phi}$ such that for each compact $K \subset \mathbb{R}^{n}$, for some $c>1$ we have

$$
\sup _{\operatorname{Re} \zeta \in K}|\bar{\partial} \tilde{\phi}| e^{g_{c}(\operatorname{Im} \zeta)}<\infty .
$$

If $\phi$ has compact support and $U$ is a complex neighborhood of supp $\phi$ we can choose $\tilde{\phi}$ with support in $U$.

For a proof, see, e.g., 2. It follows that composition of functions in $B_{h}$ stays in $B_{h}$. In a completely analogous way as before we can now define a hyperoperator $A \in H_{B_{h, 0}}(X)$ as a continuous multiplicative mapping $B_{h, 0}\left(\mathbb{R}^{n}\right) \rightarrow \mathrm{E}(X)$ such that $\cup_{\phi \in B_{h, 0}} \operatorname{Im} A(\phi)=D$ is dense and $\cap_{\phi \in B_{h, 0}} \operatorname{Ker} A(\phi)=\{0\}$. Everything that is done in Sections 3,4, and 5 carry over directly to these ultrahyperoperators; for instance, $D$ is the set of $x \in X$ such that $x=A(\chi) x$ for some cut-off function $\chi$ in $B_{h}$. If $A \in H_{B_{h, 0}}(X)$, then $\|A(\phi)\| \leq C_{c} \sup _{K_{c}^{\prime}}|\phi|_{\mathcal{A}_{c h}}$ for each $c>1$. If we 
define $\omega_{\zeta-z}^{\chi}=\left.\chi(\xi) \omega_{\zeta-\xi}\right|_{\xi=a}$ it turns out that $\left\|\omega_{\zeta-z}^{\chi}\right\| \leq C_{c} \exp g_{c}(\operatorname{Im} \zeta)$ for each $c>1$. If $\operatorname{supp} \phi \subset\{\chi=1\}$ we thus have the representation

$$
A(\phi)=\int \omega_{\zeta-a}^{\chi} \wedge \bar{\partial} \tilde{\phi}(\zeta)
$$

\section{INVARIANT SUBSPACES AND SPECTRAL DECOMPOSITION}

Precisely as for a bounded operator (tuple of commuting bounded operators) that admits a smooth functional calculus, for a hyperoperator $a$ there is a rich structure of invariant subspaces as well as spectral decompositions.

Proposition 9.1. Assume that $A \in H_{\mathcal{D}\left(\mathbb{R}^{n}\right)}(X), f \in \mathcal{E}\left(\mathbb{R}^{n}, \mathbb{R}^{m}\right)$, and let

$$
X^{\prime}=\left\{x \in D_{A} ; f(a) x=0\right\} .
$$

Then $Y=\overline{X^{\prime}}$ is an a-invariant subspace of $X$, and $a^{\prime}=\left.a\right|_{Y}$ is a hyperoperator. Moreover, $D_{a^{\prime}}=X^{\prime}$ and

$$
\text { int }\{f=0\} \cap \sigma(a) \subset \sigma\left(a^{\prime}\right) \subset\{f=0\} \cap \sigma(a) .
$$

If $\{f=0\}$ contains some open subset of $\sigma(a)$, then $Y$ has nontrivial vectors.

Proof. Since $f(a)$ and $\phi(a)$ commute, $X^{\prime}$ and hence $Y$ are $a$-invariant. If $\phi$ has compact support, then $\phi(a)$ is bounded, and hence $\phi\left(a^{\prime}\right)$ extends to a bounded operator on $\overline{X^{\prime}}$. Moreover, the continuity with respect to $\phi$ is clear. Since $1\left(a^{\prime}\right) x=x$ for all $x \in X^{\prime}$, the properties $(i)$ and (ii) in Definition 1 are satisfied, so $a^{\prime}$ is indeed a hyperoperator on the Banach space $Y$.

By definition, $X^{\prime} \subset D_{a}$. If $x \in D_{a^{\prime}}$, then $x=\chi\left(a^{\prime}\right) x$ for some $x \in Y$. This means that $x=\chi(a) x$ and so $x \in D_{a}$, and moreover $f(a) x=0$. Thus $D_{a^{\prime}}=X^{\prime}$.

If $\phi(a)=0$ for all $\phi \in \mathcal{D}(\omega)$ then $\phi\left(a^{\prime}\right)=0$ for all such $\phi$, and hence $\sigma\left(a^{\prime}\right) \subset \sigma(a)$. If $p$ is any point outside $\{f=0\}$ then $f_{j}(p) \neq 0$ for some $f_{j}\left(f=\left(f_{1}, \ldots, f_{m}\right)\right)$. We may assume that $f_{j}(p)=1$. If $\omega \ni p$ is small enough, $\left|f_{j}-1\right| \leq 1 / 2$ in $\omega$. For $\phi \in \mathcal{D}(\omega)$ we have that

$$
\phi(a) x=\phi(a)\left(1-f_{j}\right)^{N}(a) x=\left(\phi\left(1-f_{j}\right)^{N}\right)(a) x, \quad x \in X^{\prime},
$$

and since $\phi\left(1-f_{j}\right)^{N} \rightarrow 0$ in $\mathcal{D}(\omega)$ when $N \rightarrow \infty$ we can conclude that $\phi(a) x=0$. Thus $\omega$ is contained in the complement of $\sigma\left(a^{\prime}\right)$ and so we have proved the second inclusion in (9.1) To see the first one, take $p \in$ int $\{f=0\} \cap \sigma(a)$ and a neighborhood $\omega$ such that $p \in \omega \subset\{f=0\}$. Since $\omega$ intersects $\sigma(a)$ there exists some $\phi \in \mathcal{D}(\omega)$ and $z \in X$ such that $x=\phi(a) z \neq 0$. However, then $x \in D$ and $f(a) x=(f \phi)(a) z=0$ since $f \phi=0$, so $x \in X^{\prime}$. Thus $\omega$ intersects $\sigma\left(a^{\prime}\right)$. Since $\omega \ni p$ can be chosen arbitrarily small, we conclude that $p \in \sigma\left(a^{\prime}\right)$. If $\sigma\left(a^{\prime}\right)$ is nonempty, then $Y$ is nontrivial, and so the last statement follows from (9.1). 
If $p$ is an isolated point in $\sigma(a)$ and $f=0$ in a neighborhood of $p$, then $X^{\prime}$ is non-trivial. There are also non-trivial $a$-invariant subspaces as soon as $\sigma(a)$ contains more than one point. Notice that $a^{\prime}$ is bounded if $\{f=0\} \cap \sigma(a)$ is compact.

It is easy to make spectral decompositions. Let $A \in H_{\mathcal{D}\left(\mathbb{R}^{n}\right)}(X)$ be a hyperoperator and let $\left\{\Omega_{j}\right\}$ be a locally finite open cover of $\sigma(a)$. Moreover, choose $\phi_{j} \in \mathcal{E}\left(\mathbb{R}^{n}\right)$ such that $\Omega_{j} \subset\left\{\phi_{j}=1\right\}$, and let

$$
X_{j}=\left\{x \in D_{A} ; \phi_{j}(a) x=x\right\} .
$$

If $\Omega_{j}$ is bounded, we can choose $\phi_{j}$ in $\mathcal{D}\left(\mathbb{R}^{n}\right)$ and then $X_{j}=\operatorname{Ker}\left(e_{X}-\right.$ $A(\phi))$ is a closed subspace of $D_{A}$. Then $X_{j}$ are $a$-invariant subspaces, $\sigma\left(\left.a\right|_{X_{j}}\right) \subset \Omega_{j} \cap \sigma(a)$, and

$$
\sum_{1}^{\infty} X_{j}=D_{A} .
$$

All these statements but the last one follows from Proposition 9.1. To see (9.2), choose a smooth partition of unity $\chi_{j}$ subordinate to the cover $\left\{\Omega_{j}\right\}$. Then, since $\sum \chi_{j}=1$, for each $x \in D_{A}$ we have $x=\sum_{1}^{M} \chi_{j}(a) x$ for some $M$. However, $\left(1-\phi_{j}\right) \chi_{j}=0$ so $\chi_{j}(a) x$ belongs to $X_{j}$. Hence, (9.2) follows.

In general the sum (9.2) is not direct. However, if $\sigma(a)$ is a disjoint union of closed sets $F_{j}$, we can find $\phi_{j}$ with disjoint supports such that $\left\{\phi_{j}=1\right\}$ contain a neighborhood of $F_{j}$. If $x \in X_{j} \cap X_{k}$, then $x=\phi_{j}(a) x=\phi_{j}(a) \phi_{k}(a) x=\left(\phi_{j} \phi_{k}\right)(a) x=0$, and hence we get

$$
D_{A}=\oplus_{1}^{\infty} X_{j} \text {. }
$$

Example 7. Let $A \in H_{\mathcal{D}\left(\mathbb{R}^{n}\right)}(X)$ and let $f \in \mathcal{E}\left(\mathbb{R}^{n}, \mathbb{R}^{m}\right)$ be a mapping such that $f(a)=0$. From Corollary 5.5 (or (9.1)) we know that $\sigma(a) \subset\{f=0\}$. Let us also assume that the zero set $\{f=0\}=\left\{\alpha^{j}\right\}$ is discrete. Then we have the decomposition $D_{A}=\oplus_{1}^{\infty} X_{j}$ where $\sigma\left(\left.a\right|_{X_{j}}\right)=\left\{\alpha^{j}\right\}$. For each $j$, let $g_{1}^{j}, \ldots, g_{\ell_{j}}^{j}$ be functions in the local ideal generated by $f$ at $\alpha^{j}$, and let $Y_{j}=\left\{x \in D_{A} ; g_{\ell}^{j}(a) x=0, \ell=1, \ldots, \ell_{j}\right\}$. If $x \in X_{j}$, then $x=\phi_{j}(a) x$, and since moreover $g_{\ell}^{j} \phi_{j}=\sum_{k} h_{k} f_{k} \phi_{j}$ for some $h_{k}$, it follows that $x \in Y_{j}$. Thus $X_{j} \subset Y_{j}$. Furthermore, if for each $j$ the common zero set of $g_{\ell}^{j}$ is just the point $\alpha_{j}$, then by (9.1), $\sigma\left(a, \bar{Y}_{j}\right) \subset\left\{\alpha_{j}\right\}$. If $x \in Y_{j} \cap Y_{i}$, therefore $\sigma_{x}(a)=\emptyset$, and hence $x=0$ since $a$ is a hyperoperator. It therefore follows that $X_{j}=Y_{j}$.

If all zeros of $f$ are of first order, i.e., the local ideal at $\alpha^{j}$ is generated by $\xi_{i}-\alpha_{i}^{j}, i=1, \ldots, n$, then $X_{j}$ is the eigenspace

$$
X_{j}=\left\{x \in D_{A} ; a_{i} x=\alpha_{i}^{j} x, i=1, \ldots, n\right\} .
$$

If $A \in H_{\mathcal{D}(\mathbb{C})}(X)$ and $f$ is holomorphic in a neighborhood of $\sigma(a)$ and the zeros $\alpha^{j}$ have multiplicities $r_{j}$, then $X_{j}=\left\{x \in D_{A} ;\left(a-\alpha_{j}\right)^{r_{j}} x=\right.$ $0\}$. 
The situation in this example appears naturally when we consider homogeneous solutions to an equation like $f(a) x=0$.

Example 8. Let $\mathcal{A}_{h}\left(\mathbb{R}^{n}\right)$ be a Beurling algebra, cf., Section 8 , containing cut-off functions, and let $X$ be the space of inverse Fourier transforms of the dual space $\mathcal{A}_{h}^{\prime}$. Then the tuple of commuting operators $a_{j}=i \partial / \partial \xi_{j}$ on $X$ admits an $\mathcal{A}_{h}$ functional calculus (since $\mathcal{A}_{h}$ is an algebra). Then $D_{a}$ is the space of (inverse) Fourier transforms of elements with compact supports in $\mathcal{A}_{h}^{\prime}$. Notice that $\mathcal{A}_{h}^{\prime}$ contains all distributions with compact support, but also some hyperfunctions of infinite order. Let $f$ be a $\mathcal{A}_{h^{-}}$ smooth mapping and consider the space $\left\{x \in D_{a} ; f(a)=0\right\}$. If $x$ is the inverse Fourier transform of $u$, then $f(t) u(t)=0$, which means that $u$ has support on $Z=\left\{t \in \mathbb{R}^{n} ; f(t)=0\right\}$. It follows that we have the representation

$$
x(\xi)=\int_{\mathbb{R}^{n}} e^{i \xi \cdot t} u(t) d t,
$$

meaning the action of $u$ on $t \mapsto \exp i \xi \cdot t$. Since $u$ has support on the set $Z=\{f=0\}, x$ is expressed as a combination of exponentials with frequencies in $Z$.

Even if $f$ is a polynomial, only solutions generated by real frequencies can appear as long as we have restricted to non-quasianalytic classes. To get an operator-theoretic frame of this kind for the general fundamental principle of Ehrenpreis and Palamodov, 9] and [14, one must consider operators that only admit a holomorphic functional calculus.

\section{Non-COMMUTING HYPEROPERATORS}

Assume that $A_{1}, \ldots, A_{m}$ are in $H_{\mathcal{D}\left(\mathbb{R}^{n_{j}}\right)}$ but not necessarily commuting. Then we can form the tensor product $A=A_{1} \otimes \cdots \otimes A_{m}$, and obtain a linear continuous, though not multiplicative, operator $\mathcal{D}\left(\mathbb{R}^{n}\right) \rightarrow \mathrm{E}(X)$, where $n=n_{1}+\cdots+n_{m}$. This can also be done explicitly by the formula (6.7). We also write this operator of course as $\phi\left(a_{1}, \ldots, a_{m}\right)$. In case when all $n_{j}=1$ and $a_{j}$ are HS operators, we get back the definition in [3]. Now the order of the operators is crucial. Therefore it is convenient to use Feynman notation, see, e.g., 13. Then this operator $\phi\left(a_{1}, \ldots, a_{m}\right)$ can be written $\phi\left(a_{1}^{m}, \ldots, a_{m}^{1}\right)$ indicating that the operator $a_{m}$ is to be applied first, then $a_{m-1}$ etc and finally $a_{1}$, and the order is reflected by the order of the resolvents. Therefore, if $b$ is a bounded operator one can easily define for instance

$$
\phi\left(\stackrel{3}{a_{1}}, \stackrel{1}{a_{2}}\right) \stackrel{2}{b}=\iint\left(\partial_{\overline{\zeta_{1}}} \partial_{\overline{\zeta_{2}}}\right) \tilde{\phi}\left(\zeta_{1}, \zeta_{2}\right) \wedge \omega_{\zeta_{1}-a_{1}}^{\chi_{1}} \wedge b \omega_{\zeta_{2}-a_{2}}^{\chi_{1}} .
$$

Notice that this is not an ordinary composition of $f\left(\stackrel{3}{a_{1}}, a_{2}\right)$ and $b$, while for instance $f\left(\stackrel{2}{a}, \stackrel{1}{a_{2}}\right) \stackrel{3}{b}=b f\left(\stackrel{2}{a}, \stackrel{1}{a_{2}}\right)$. 


\section{REFERENCES}

[1] M. Andersson: (Ultra)differentiable functional calculus and current extension of the resolvent mapping, Ann. Inst. Fourier, 53 (2003), 903-926.

[2] M. Andersson \& B. Berndtsson: Non-holomorphic functional calculus for commuting operators with real spectrum, Ann. Scuola Norm. Sup. Pisa, 1 (2002), 925-955.

[3] M. Andersson \& J. SuÖstrand: Functional caclulus for non-commuting operators with real spectra via an iterated Cauchy formula, J. Functional Anal., 210 (2004), 341-375.

[4] A. Beurling: On quasianalyticity and general distributions, Lecture notes, Stanford (1961).

[5] M. Dimassi \& J. SJÖstrand: Spectral asymptotics in the semi-classical limit, London Math. Soc. LNS 268, Cambridge Univ. Press, 1999.

[6] B. Droste: Extension of analytic functional calculus mappings and duality by $\bar{\partial}$-closed forms with growth, Math. Ann. 261, 185-200 (1982).

[7] E.M. Dynkin: An operator calculus based on the Cauchy-Green formula. (Russian), Investigations on linear operators and the theory of functions, III. Zap. Nauv cn. Sem. Leningrad. Otdel. Mat. Inst. Steklov. (LOMI) 30 (1972), 33-39..

[8] J. Eschmeier \& M. Putinar: Spectral Decompositions and Analytic Sheaves, Clarendon Press, Oxford 1996.

[9] Ehrenpreis, Leon: Fourier analysis in several complex variables, Pure and Applied Mathematics, Vol. XVII Wiley-Interscience Publishers A Division of John Wiley \& Sons, New York-London-Sydney 1970.

[10] B. HelfFer \& J. SJÖSTRAND: Equation de Schrödinger avec champ magnétique et équation de Harper, Springer Lecture Notes in Physics 345(1989), 118-197.

[11] L. HÖRMANDER: The analysis of linear partial differential operators, I-IV, Grundlehren, Springer, 256, 257, 274, 275, 1983-1985.

[12] E.-J. IonaşCU \& F.-H. VASIlescu: Joint spectral properties for permutable linear transformations, J. Reine Angew. Math., 426 (1992), 23-45.

[13] V.E. Nazaikinski \& V.E. Shatalov \& B.Yu. Sternin: Methods of noncommutative analysis, de Gruyter Studies in Math. 22, Berlin, New York 1996.

[14] V.P. Palamodov: Linear differential operators with constant coefficients, Die Grundlehren der mathematischen Wissenschaften, Band 168 SpringerVerlag, New York-Berlin 1970.

[15] H. Samuelsson: Multidimensional Cayley transforms and tuples of unbounded operators, Preprint Gothenburg (2004).

[16] S. SANDBERG: On non-holomorphic functional calculus for commuting operators, Math. Scand., 93 (2003), 109-135.

[17] K. SchmÜDGen: On commuting unbounded self-adjoint operators I, Acta Sci. Math. 47, (1984), 131-146.

[18] K. SChmüDGEN: On commuting unbounded self-adjoint operators III, Manuscripta Math., 54, (1985), 221-247.

[19] K. SChmüDgen: On commuting unbounded self-adjoint operators IV, Math. Nachr., 125, (1986), 83-102.

[20] K. SChmüdgen \& J. FrIEdRICH: On commuting unbounded self-adjoint operators II, J. Integral Equ. and Operator Theory, 7, (1984), 815-867.

[21] J.L. TAYLOR: A joint spectrum for several commuting operators, J. Funct. Anal. 6 (1970), 172-191. 
[22] F-H Vasilescu: Analytic Functional Calculus and Spectral Decomposition, Kluwer Acad. Publ. (1982).

[23] F-H VAsIlescu: Joint spectral properties for pairs of permutable selfadjoint transformations, Linear operators in function spaces (Timişoara, 1988), Oper. Theory Adv. Appl., 43, Birkhäuser, Basel, (1990), 313-321.

[24] F-H VAsilescu: Quaternionic Cayley transform, J. Funct. Anal., 164, (1999), 134-162.

Department of Mathematics, Chalmers University of Technology AND the UnIVERsity of GÖTEBorg, S-412 96 GÖTEBORG, SWEDEN

E-mail address: matsa@math.chalmers.se 